\title{
INDEX OF FOSSILIFEROUS LOCALITIES OF THE TŘENICE FORMATION (LOWER ORDOVICIAN OF THE PRAGUE BASIN, CZECH REPUBLIC)
}

\author{
Jaroslav Kraft ${ }^{\dagger}$, Michal Mergl1, Tomáš Hroch² \& Petr Kraft ${ }^{3,4}$ \\ ${ }^{1}$ Center of Biology, Geosciences and Environmental Sciences, Faculty of Education, University of West Bohemia in Plzeň, \\ Klatovská 51, 30619 Plzeň, Czech Republic; E-mail: mmergl@cbg.zcu.cz \\ 2 Czech Geological Survey, Klárov 3, 11821 Praha 1, Czech Republic; E-mail: tom.hroch@gmail.com \\ ${ }^{3}$ Institute of Geology and Palaeontology, Faculty of Science, Charles University in Prague, Albertov 6, 128 43 Praha 2, Czech \\ Republic; E-mail: kraft@natur.cuni.cz \\ ${ }^{4}$ West Bohemian Museum in Plzeň, Kopeckého sady 2, 30100 Plzeň, Czech Republic
}

\begin{abstract}
Twenty nine fossiliferous localities of the Třenice Formation are described. As we were able to find they represent all fossil sites ever been described from that basal unit of the Prague Basin. Based on critical evaluation of the previously published data fossil taxa from all relevant publications are summarized and historic names used for the localities are mentioned. Updated lists of fauna are compiled for each locality as well as for the formation as a whole.
\end{abstract}

Key words: Ordovician, Třenice Formation, Prague Basin, fossils

\section{INTRODUCTION}

Before he passed away, the senior author compiled a large data set on the Lower and Middle Ordovician of the Prague Basin (Barrandian area, Bohemia, Czech Republic). As a worker of the West Bohemian Museum in Plzeň; and prior to that at the Museum of Dr. B. Horák in Rokycany; his primary focus was on fossil sites in the south-western part of the Basin. Later in his life, he extended his scope of interest with one of the co-authors (P. K.), of this paper, to include the entire Prague Basin regardless of the age of sediments. This effort culminated in 2001-2003 when they gathered complex data on fossiliferous localities of the Prague Basin under the project of Ministry of Culture. As these important data have not had wide distribution a series of papers is intended to make the key data available; this being the first in the series. It is hoped that this project will serve as a basic dataset for diverse research in the Ordovician of the Barrandian area. It is also an explanatory document in which synonymous names for localities are listed to aid the researcher when using older publications and collections in their research. This, the first contribution, is focused on the Třenice Formation, the basal lithostratigraphic unit of the Prague Basin.
The Třenice Formation is the basal volcanosedimentary fill in the Prague Basin (Havlíček 1981, 1998). It is generally coarser-grained sandstones and conglomerates often with the volcanic admixture (Kukal 1961, Hroch et al. 2012).

First fossils associated with the iron ore mining were studied in 19th century. Paleontologic fieldwork oriented on other localities was begun in the 20th century by C. Klouček, J. Koliha, and R. Růžička. Later, the Třenice Formation brachiopods (e.g. Havlíček 1982a, Mergl 2002) and trilobites (e.g. Vaněk 1965, Mergl 2006) have been mostly studied because they are the dominant groups. They were also included in general studies on fossil associations and their aspects (Havlíček 1982b, Havlíček \& Fatka 1992, Havlíček \& Vaněk 1966). Recently, special studies were focused on stratigraphic consequences of the succession of fossil assemblages (Mergl 2009), taphonomic aspects (Mergl 1996, 1997c) and peculiar community (Lehnert et al. 2004) in the Třenice Formation.

\section{LOCALITIES}

Only the Třenice Formation localities which are known to have yielded or, in some cases, possibly yeilded the fauna are included in the list. Records 


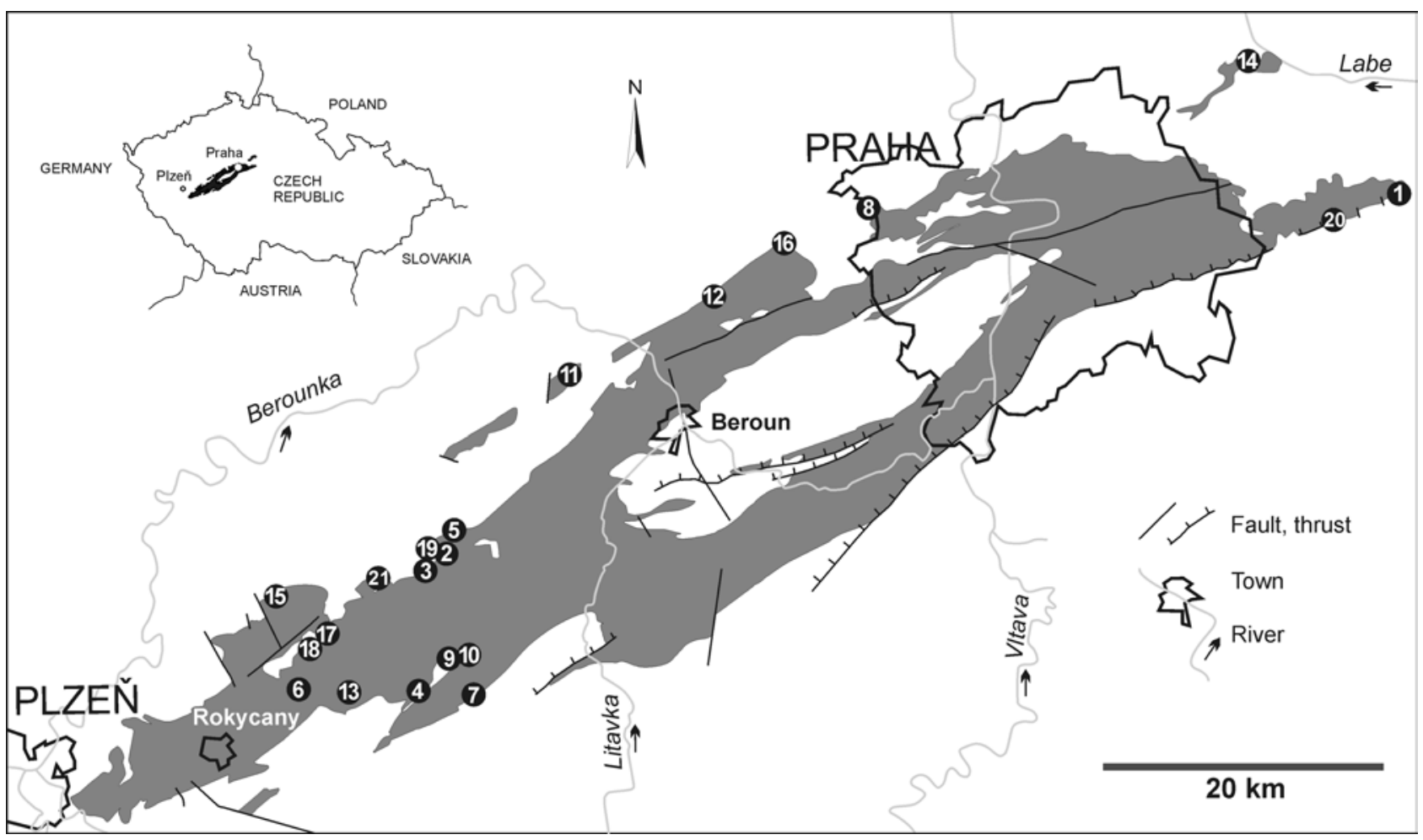

Figure 1. Sketch map of the Prague Basin relic and positions of the described localities. Location of the Prague Basin (black) in the Czech Republic (left up). The localities are ordered the same as in the text; those situated close to each other are plotted as a single point. 1 - Břzzany - "Na Chrástnici" Quarry; 2 - Cerhovice - Cerhovská hora Hill; 3 - Cerhovice Kvásek Hill; 4 - Cheznovice - Žlebec; 5 - Drozdov - Holý vrch Hill, Drozdov - Opiš; 6 - Holoubkov - Ouzký; 7 - Horní Kvaň field; 8 - Hostivice; 9 - Jivina - quarries, Jivina Hill; 10 - Kleštěnice - Jalový potok Brook; 11 - Krušná hora - Gabriela Mine, Krušná hora - gallery, Krušná hora Hill; 12 - Libečov - Na močidle, Libečov - gallery; 13 - Medový Újezd, Medový Újezd - Hradiště; 14 - Popovice - Marešovka Quarry; 15 - Skomelno - Na Solích; 16 - Svárov; 17 - Těškov - Kněžský vrch Hill (quarry); 18 - Těškov - north-west of the village; 19 - Týček; 20 - Úvaly - shaft, Úvaly; 21 - Zbiroh - Bukov (old dump), Zbiroh - Bukov (quarries).

with insufficiently documented localities and lists of ambiguous fossils are ommitted. This approach significantly impacts old papers in which fossils from several formations were listed as a single assemblage.

Localities are listed in alphabetical order and their descriptions are structured as follows: brief geographical location (geographic co-ordinates if available), lithology, general remarks, references, and updated revised taxonomic list. The references are ordered chronologically. The original locality name or names used in a publication follows the authors, the original list of taxa (including author and year, and errors; original letter style is ignored and italics are used for latin in modern way) is compiled. Translations to English are placed in square brackets for the localities with obscure or difficult names. Original Czech or German descriptions of fossils are supplemented with verbatim English translations in square brackets. These reports are important in illustrating the history, the available taxa, as currently identified, for systematic studies. Old papers written in Czech, French or German will now have English translations of pertinent parts.

The last section below is a complete updated list of that in Havlíček \& Vaněk (1966).

Institutional abbreviations:

MR - Museum of Dr. B. Horák in Rokycany,

NM - National Museum in Prague,

PCZCU - Faculty of Education, University of West Bohemia. 


\section{LIST OF LOCALITIES}

\section{Břežany - "Na Chrástnici” Quarry}

Geography: Large abandoned quarry 700 m eastnorth-eastern of the village of Břežany II (distance and direction related to the church in the centre of the village), $\sim 27 \mathrm{~km}$ east of Prague (measured from the centre of the city). Protected area PP Chrástnice. (Coordinates read from map: $\mathrm{N} 50^{\circ} 05^{\prime} 44.5^{\prime \prime}$ E $14^{\circ} 48^{\prime} 54.5^{\prime \prime}$ for centre of the quarry). Cadastre of Břežany II, District of Kolín.

Lithology: Pale yellow-grey lithic sandstone with chert nodules, shale intercalations.

Remarks: Steep slopes of the quarry covered by vegetation has only poor outcrops of the fossiliferous layers at present. Thin shale bed with dendroids has not been accessible for over 80 years. The fossil assemblage differs from other assemblages of the Třenice Formation by the abundance of well preserved but tectonically deformed fossils. That is way some authors assumed this locality to be equivalent of the Mílina Formation.

Koliha (1926a, 1926b): Břežany, Břežany u Českého Brodu [Břežany near Český Brod].

Dictyograptus flabelliformis (Eichwald)

forma, která upomíná Dictyograptus flabelliformis (Eichw.) var. norvegica Kjerulf [type resembling Dictyograptus flabelliformis (Eichw.) var. norvegica Kjerulf]

staurograpti [staurograptids]

Obolus Eichw.

Lingulella Salt.

snad i Orbiculoidea d'Orb. [probably Orbiculoidea d'Orb.]

Heritsch (1928): Břežany.

Obolus Feistmanteli

Lingulella expulsa

Obolus

Dictyograptus flabelliformis Eichw. Varietät norvegica Kjerulf

Stomatograptus

Obolus aus dem Subgenus Schmidtia [Obolus (Schmidtia)]

Klouček (1931a, 1931b): Břežany.

Dictyonema flabelliforme Eichw.

Obolus siluricus Eichw. (Ob. cf. Barrandei)

orthisky [orthids]

Lingulella sp.

Havlíček (1949a): Břežany.

Tritoechia kolihai n. sp.
Prantl \& Přibyl (1949b): Břežany - lom vých. od obce [Břežany - quarry east of the village]; Břežany.

Dictyonema flabelliforme intermedium nov. subspec. Callograptus kodymi nov. spec.

?Desmograptus sp.

Havlíček (1950): Chrástnice u Břežan [Chrástnice near Břežany].

Dictyonema flabelliforme intermedium Prantl a Přibyl

Callograptus kodymi Prantl a Přibyl

?Desmograptus $\mathrm{sp}$.

Thysanotos barrandei barrandei (Klouček)

Thysanotos barrandei primus (Koliha)

Lingulobolus feistmanteli minor Prantl a Růžička

Lingulella cf. insons (Barr.)

Obolus giganteus Koliha

Tritoechia kolihai Havlíček

Havlíček (1951): Břežany.

Tritoechia kolihai Havlíček, 1949

Kraft (1975): Břežany - quarry „Na Chrástnici“. Dictyonema flabelliforme intermedium Prantl et Přribyl, 1949

Callograptus kodymi Prantl et Přibyl, 1949

Havlíček (1977): Břežany, quarry on Chrástnice Hill. Protambonites kolihai (Havlíček, 1949)

Havlíček (1982a): Břežany, Chrástnice Hill (quarry); Břežany.

Thysanobolus lingulides sp. $\mathrm{n}$.

Thysanobolus giganteus (Koliha, 1937)

Mergl (1997b): Břežany ("Na Chrástnici" quarry); Břežany (old quarry); Břežany (abandoned quarry "Na Chrástnici" East of Prague).

Thysanotos primus (Koliha, 1924)

Kraft (1997): Břežany - lom "Na Chrástnici" [Břežany - "Na Chrástnici" quarry].

Dictyonema intermedium Prantl et Přibyl, 1949

Callograptus kodymi Prantl et Přibyl, 1949

Mergl (2002): Břežany II (Na Chrástnici quarry); Břežany II (lom "Na Chrástnici" - "Na Chrástnici" quarry).

Thysanotos primus (Koliha, 1924)

Updated list of fauna:

Leptembolon sp.

Thysanotos primus (Koliha, 1924)

Protambonites kolihai (Havlíček, 1949)

Dictyonema intermedium Prantl et Přibyl, 1949

Callograptus kodymi Prantl et Přibyl, 1949 


\section{Cerhovice - Cerhovská hora Hill}

Geography: Old filled quarries, and small natural outcrops in the eastern and south-eastern slopes of the Cerhovská hora Hill (also called Třenická hora Hill) near the village of Cerhovice, $\sim 1 \mathrm{~km}$ northwest of the centre of village, western of Trrenice. Cadastre of Cerhovice, District of Beroun.

Lithology: Predominantly greenish and brownish lithic cross-bedded sandstones. Several, less resistant lenticular volcanigenic sandstones 10 to 15 centimeters thick are intercalacted in the crossbedded sandstones. The volcanigenic sandstones are characterized by a large fraction of non-vesiculated chloritized volcanic glass and microcrystalline volcanic rocks (Hroch et al. 2012).

Remarks: Large quarries have limited access at present time, sandstones are poorly exposed. Exact stratigraphic levels of fossils are unknown. Fossils are generally rare.

? Lipold (1863): Umgebung von Cerhowice [vicinity of Cerhovice].

Lingula Feistmantelli Barr.

Barrande (1879): Czerhowitz.

Lingula Feistmanteli. Barr.

Jahn (1904a): Cerhovice (Třenice)

Obolella Feistmanteli

Jahn (1904b): Cerhovic (it is not specified and the locality is deduced from the previous record)

Obolella Feistmanteli

Klouček (1920a): Cerhovská hora; lomy na Cerhovské hoře [quarries at Cerhovská hora Hill]. brachiopod blízký menším varietám druhu Obolus Feistmanteli Barr. [brachiopod similar to smaller varieties of the species Obolus Feistmanteli Barr.] neobyčejně velká acrotreta [unusually large Acrotreta]

Koliha (1924): Cerhovská hora.

Obolus (Lingulobolus) Feistmanteli (Barr.)

Andrusov (1925): Cerhovská hora.

Orthis Kettneri Klou.

Kraft (1928): Cerhovská hora; Cerhovická hora. Obolus Feistmanteli

Havlíček (1982a): Cerhovická hora near Cerhovice; Cerhovice.

Hyperobolus feistmanteli (Barrande, 1879)
Mergl (2002): Cerhovice; Cerhovice (Cerhovská hora Hill); Cerhovice (Cerhovický vrch Hill); Cerhovice (Cerhovská hora - Cerhovská hora Hill). Hyperobolus feistmanteli (Barrande, 1879)

Updated list of fauna:

Hyperobolus feistmanteli (Barrande, 1879)

\section{Cerhovice - Kvásek Hill}

Geography: Old quarries in the Kvásek Hill $\sim 3 \mathrm{~km}$ west of the centre of the village of Cerhovice, $2.2 \mathrm{~km}$ south-west of the church at the town square in Zbiroh. Cadastre of Cerhovice, District of Beroun and Cadastre of Zbiroh, District of Rokycany.

Lithology: Greenish, medium to coarse-grained lithic cross-bedded sandstone.

Remarks: Exact fossil sampling sites are uknown.

Jahn (1904a): Cerhovice (Kvásek)

Obolella Feistmanteli

Klouček (1920a): Kvásek.

Ob. Feistmanteli (typus)

Koliha (1924): Kvásek.

Obolus (Lingulobolus) Feistmanteli (Barr.)

Lingulella expulsa (Barr.)

? Heritsch (1928): Třenice.

Obolus Feistmanteli

Lingulella expulsa

Havlíček (1982a): Kvásek near Cerhovice. Hyperobolus feistmanteli (Barrande, 1879)

Mergl (2002): Kvásek Hill; Cerhovice (Kvásek Hill).

Expellobolus expulsus (Barrande, 1879)

Hyperobolus feistmanteli (Barrande, 1879)

Updated list of fauna:

Expellobolus expulsus (Barrande, 1879)

Hyperobolus feistmanteli (Barrande, 1879)

\section{Cheznovice - Žlebec}

Geography: Quarry and wooded dumps of former iron ore mine in Žlebec near prominent curves of the road Strašice-Olešná, $1.1 \mathrm{~km}$ south-east-south of chapel in the village of Cheznovice (N $49^{\circ} 46^{\prime} 13.1^{\prime \prime}$, E $\left.13^{\circ} 47^{\prime} 33.3^{\prime \prime}\right)$. Cadastre of Cheznovice, District of Rokycany.

Lithology: Volcanigenic clastics: sandstone and fine-grained conglomerate (with granules up to $0.5 \mathrm{~cm}$ in diameter). The clasts consist primarily 
of rhyolite, andesite, and non-vesiculated chloritized volcanic glass fragments.

Remarks: Name Žlebec has been used for several fossil sample sites. The first is abandoned and by waste material partly infilled large quarry east of the road (Plate VII, Fig.1). This is likely the locality referred by Koliha (1924), Kraft (1928), and Havlíček (1982a). Another locality is situated westward (N 49 $\left.46^{\prime} 12.0^{\prime \prime}, \mathrm{E} 13^{\circ} 47^{\prime} 39.0^{\prime \prime}\right)$ on a low slope of a small quarry near pits and dumps of the abandoned mines. Poorly outcropping sandstone beds yielded a distinct fauna described by Mergl $(2002,2006,2009,2012)$. The name Žlebec is also used for large dump and test pit located more to northwest. There, the haematitised tuffs of the Klabava Fomation and cherts of the Mílina Formation with a depauperate fauna of lingulate brachiopods were mined.

Koliha (1924): Žlebec u Cheznovic [Žlebec near Cheznovice].

Obolus (Lingulobolus) Feistmanteli (Barr.)

Kraft (1928): Žlebec u Cheznovic; veliký lom v lese jižně od Cheznovic (u k. 522) [large quarry in the forest south of Cheznovice (near elevation point 522)].

Obolus Feistmanteli

Havlíček (1982a): Žlebec near Cheznovice. Hyperobolus feistmanteli (Barrande, 1879)

Mergl (2002): Cheznovice (Žlebec); Cheznovice (Žlebec, old dump).

Rosobolus magnus sp. $\mathrm{n}$.

Orbithele discontinua Mergl, 1981

Acrotreta grandis Klouček, 1919

Siphonobolus simulans (Růžička, 1927)

Mergl (2006): Cheznovice; Cheznovice (“Žlebec”). Holoubkocheilus granulatus (Růžička, 1926)

Mergl (2009): Žlebec u Cheznovic [Žlebec near Cheznovice]; Cheznovice.

trilobiti [trilobites]

Acrotreta grandis Klouček

Siphonobolus simulans (Růžička)

Orbithele discontinua Mergl

Mergl (2012): Cheznovice.

Rosobolus magnus Mergl, 2002

Leptembolon insons (Barrande, 1879)

Dactylotreta sp.

Acrotreta sp.
Siphonobolus simulans (Růžička, 1927)

Apheoorthina sp.

Eoorthis? sp.

Trilobita gen. et sp. indet.

Updated list of fauna:

Leptembolon insons (Barrande, 1879)

Rosobolus magnus Mergl, 2002

Acrotreta sp.

Dactylotreta sp.

Siphonobolus simulans (Růžička, 1927)

Apheoorthina sp.

Eoorthis? sp.

Trilobita gen. et sp. indet.

\section{Drozdov - Holý vrch Hill}

Geography: Old small abandoned quarries in the south slope of the Holý vrch Hill $\sim 1.4 \mathrm{~km}$ north of the centre of the village of Drozdov. Cadastre of Drozdov, District of Beroun.

Lithology: Greenish lithic sandstone.

Remarks: Small quarries yielded nicely preserved internal and external moulds of brachiopods. Judging from stored samples, the fossils were abundant in particular levels. At present, exact positions of fossiliferous layers are uknown.

Koliha (1924): Holý vrch u Drozdova [Holý vrch Hill near Drozdov].

Obolus (Lingulobolus) Feistmanteli (Barr.)

Lingulella expulsa (Barr.)

Kraft (1928): Holý vrch u Drozdova [Holý vrch Hill near Drozdov]; lom na východním svahu [quarry on the eastern slope].

Obolus Feistmanteli

Orbiculoidea sodalis

Mergl (1981): Holý vrch Hill at Třenice; Holý vrch at Třenice.

Orbithele secedens (Barrande, 1879)

Havlíček (1982a): Holý vrch near Drozdov; Holý vrch. Hyperobolus feistmanteli (Barrande, 1879)

Mergl (2002): Drozdov (Holý vrch Hill). Hyperobolus feistmanteli (Barrande, 1879)

Orbithele secedens (Barrande, 1879)

Updated list of fauna:

Expellobolus expulsus (Barrande, 1879)

Hyperobolus feistmanteli (Barrande, 1879)

Orbithele secedens (Barrande, 1879) 


\section{Drozdov - Opiš}

Geography: Old small abandoned quarries in the south slope of the Opiš Hill (or Opyš Hill) 1.6 km north-east of the centre of the village of Drozdov. Cadastre of Drozdov, District of Beroun. Lithology: Greenish lithic sandstone.

Koliha (1924): Obiš u Drozdova [Obiš Hill near Drozdov].

Obolus (Lingulobolus) Feistmanteli (Barr.) Lingulella expulsa (Barr.)

Havlíček (1982a): Obiš near Drozdov; Obiš. Hyperobolus feistmanteli (Barrande, 1879)

Mergl (2002): Drozdov (Obiš Hill).

Expellobolus expulsus (Barrande, 1879)

Hyperobolus feistmanteli (Barrande, 1879)

Updated list of fauna:

Expellobolus expulsus (Barrande, 1879)

Hyperobolus feistmanteli (Barrande, 1879)

\section{Holoubkov - Ouzký}

Geography: Old shallow pit iron mine, number of small pits located in the currently wooded area north-west of the village of Holoubkov. This area is $\sim 1 \mathrm{~km}$ from the centre of the village and it is crossed by the freeway D5. The fossils were collected exclusively from the mine dumps, with the most fossiliferous samples in dumps centrered around the deepest pit (N $\left.49^{\circ} 46^{\prime} 50.2^{\prime \prime}, \mathrm{E} 13^{\circ} 40^{\prime} 47.2^{\prime \prime}\right)$. Cadastre of Holoubkov, District of Rokycany.

Lithology: Gradded conglomerate with hematite matrix (ferrolith), and finely banded haematites. Well-rounded clasts are represented by polycrystalline quartz, Neoproterozoic cherts, and rhyolites and andesites of the Křivoklát-Rokycany Complex. Remarks: The locality has been known over 150 years, but the exact stratigraphic succession of the fossil associations is uknown due to lack of exposures. However, the fossils surely came from several levels. The basal or intercalated conglomeratic bed(s) yielded encrusting problematic fossil Marcusodiction. Coeval or slightly younger association has many large rhynchonelliformean brachiopods (Poramborthis, Eoorthis, Roberorthis), abundant cystoids while the linguliformean brachiopods, and trilobites are rare. Probably the youngest assemblage of the Třenice Formation have a rich trilobite fauna, different rhynchonelliformean brachiopods (e.g. Jivinella), different cystoids, a gastropod, and a rich suite of generally small-sized linguliformean brachiopods. Abundance of laminated haematites represents an outstanding phenomenon of a stromatolitic sea-floor. At present there are very few loose blocks of fossiliferous haematites in the dumps. However, if discovered they yield abundant and well preserved fossils. Abrasion of shells indicates the littoral environment.

Lipold (1863): Auskyer Zeche bei Holoubkau [Ausky Mine near Holoubkov].

Orthis desiderata Barr.

ein ander gross noch nicht bestimmten Orthis-Art [other different large, uncertain species of Orthis]

Feistmantel (1878): Ausker Zeche bei Holoubkov [Ausky Mine near Holoubkov].

mehrere Arten von Orthis und Cystideen [many species of Orthis and cystoids]

Barrande (1879): Ausken Zeche près Holaubka [Ausky Mine near Holoubkov]; Mines de fer près Holaubka; [Iron mines near Holoubkov]; Minerai de fer près de Holoubka [Iron ore near Holoubkov]. Orthis Grimmi. Barr.

Orthis potens. Barr.

Orthis soror. Barr.

Waagen (1887): Holoubka; Ausken Zeche, Holoubka [Ausky Mine, Holoubkov].

Echinosph.? concomitans. Barr. [Echinosphaerites? concomitans. Barr.]

Echinosph. ? ferrigena. Barr. [Echinosphaerites? ferrigena. Barr.]

Krejčí \& Feistmantel (1890): důl „Ouzký” u Holoubkova ["Ouzký" Mine near Holoubkov].

Orthis Grimmi

Orthis soror

Orthis potens

Katzer (1892): Eisenerzgrube bei Holoubkau [Iron ore mine near Holoubkov], Ouzkýzeche bei Holoubkau [Ouzký Mine near Holoubkov]

Orthis Grimmi Barr.

Orth. soror

Orth. potens

Klouček (1924): Ouzký; Ouzký u Holoubkova [Ouzký near Holoubkov].

Euloma

Apatocephalus

Olenid n. sp. I.

Olenid n. sp. II (zcela nový druh) [Olenid n. sp. II (entirely new species)]

Symphysurus

Hemibarrandia (?) 
Cheirurus

Amphion (?)

asi 4 druhy billingsell (othis) mezi nimi známá Billingsella incola Barr. [some 4 species Billingsella (Orthis), known Billingsella incola Barr. among them]

velká forma acrotrety [large form of Acrotreta]

Orbiculoidea undulosa Barr. i druhá neundulosní

[Orbiculoidea undulosa Barrande and also another non-undulose]

několik zcela nových menších druhů obolů blízkých rodu Obolus Feistmanteli i druhu Ob. Barrandei Klou. [several completely new smaller species of Obolus close to the genus Obolus Feistmanteli as well as species Ob. Barrandei Klou.]

Obolus complexus Barr.

fragmenty $\mathrm{z}$ různých cystid [fragments of various cystids]

conularie [conulariid]

Klouček (1925): Ouzký; Ouzký u Holoubkova [Ouzký near Holoubkov].

Olenid n. sp. I.

Olenid n. sp. II.

Euloma sp.

Euloma (?) n. sp.

Apatocephalus sp.

Parabolinella (?)

neznámé pygidium [unknown pygidium]

Lichas sp.

Symphysurus sp. I.

Symphysurus sp. II („Hemibarrandia“).

Cheirurus sp.

Niobe (?)

Megalaspis (?)

Orthis (Billingsella) incola Barr.

Orthis Grimmi Barr, nejméně dvě variety [at least two varieties]

Orthis potens Barr.

Orthis soror Barr.

Orthis sp.

Brachiopod n. sp.

Brachiopod n. sp.

Discina (Orbiculoidea) undulosa Barr. a její varieta neundulosní [Discina (Orbiculoidea) undulosa Barr. and its non-undulose variety]

Acrotreta sp.

Acrotreta n. sp.

Obolus Barrandei (Ob. Feistmanteli var. Barrandei dle J. Kolihy) [Obolus Barrandei (Ob. Feistmanteli var. Barrandei according to J. Koliha)]

Obolus Kloučeki (?) Koliha

Obolus (?) n. sp. asi 2 var. [probably 2 varieties]
Obolus complexus var.

Obolus n. sp. (?)

Lingulella insons Barr.

Lingulella (?) n, sp.

Conularia sp.

Cystidey, nejméně 4 druhy [at least 4 species]

Problematica (deduced from the species numbering three problematic fossils are supposed)

Růžička (1926): Holoubkov (v Ouzkém) [Holoubkov (In Ouzký)]; bývalé doly v Ouzkém u Holoubkova [former mines in Ouzký near Holoubkov]; rudné ložisko v Ouzkém [ore deposit in Ouzký]; Ouzký (variants of the locality name are used only in the introduction).

Euloma granulatum n. sp.

Euloma mitratum n. sp.

Olenus (Cyclognathus?) sp. I

Olenus sp. II

Niobe ferrigena $\mathrm{n}$. $\mathrm{sp}$.

Niobe sp.

Asaphid (Megalaspis?)

Nileus Holoubkovensis n. sp.

Symphysurus Bröggeri n. sp.

Cheirurus Perneri n. sp.

Cheirurus sp.

Cyrtometopus bohemicus n. sp.

Pliomera sp.

Pliomera sp.

Lichas Kloučeki n. sp.

Lichas sp.

Lichas sp.

Illaenus (Bumastus?) sp.

Hypostom (Megalaspis?)

Hypostom (Olenid)

Klouček (1927a, 1927b): Ouzký.

Critical remarks to Růžička's (1926) descriptions can be summarized as follows:

Euloma granulatum n. sp. is valid

Euloma mitratum n. sp. is conspecific with E. granulatum

Symphysurus Bröggeri n. sp. is conspecific with S. bohemicus Klouček

Cheirurus Perneri n. sp. does not belong to the genus Cheirurus

Růžička (1927): Holoubkov (v Ouzkém) [Holoubkov (In Ouzký)]; rudní ložisko u Holoubkova (v Ouzkém) [ore deposit near Holoubkov (In Ouzký)]; Ouzký. Micromitra (Iphidella) Walcotti n. sp.

Obolus (Lingulobolus) cf. Feistmanteli (Barr.)

Obolus sp.

Obolus siluricus Eichw. 
Lingulella insons (Barr.)

Lingulella Bukovensis Kol.

Kutorgina Kolihai n. sp.

Siphonotreta Krafti n. sp.

Siphonotreta simulans n. sp.

Acrotreta grandis [Klou.]

Orbiculoidea socialis var. undulosa (Barr.)

Orthis (Billingsella) Grimmi Barr.

Orthis (Billingsella) Grimmi Bar. var. lamellosa

n. var.

Orthis (Billingsella?) sp.

Orthis (Billingsella) incola Barr.

Orthis soror Barr.

Orthis sp.

Sphaeronites crateriformis $\mathrm{n}$. sp.

Sphaeronites Batheri n. sp.

Sphaeronites sp.

Cystidea (blíže neurčitelná) [Cystidea (undeterminable more precisely)]

Clisospira sp.

Ostracod?

Heritsch (1928): Ouzký.

Oleniden [olenids]

Euloma

Cheirurus

Amphion

Lichas

Symphysurus

Apatocephalus

Parabolinella

Niobe

Kraft (1928): Úzký u Holoubkova [Úzký near Holoubkov].

Olenus

Euloma

Symphysurus sp.

Symphysurus - Hemibarrandia

Cheirurus sp.

Lichas sp.

Niobe

Nileus

celkem asi 13 druhů trilobitů [altogether about 13 species of trilobites]

Orthis (Billingsella) incola Barr.

Orthis (Billingsella) Grimmi Barr.

Orthis (Billingsella) potens Barr.

Orbiculoidea (Discina) undulosa

Acrotreta n. sp.

Acrotreta n. sp.

Obolus Feistmanteli - var. Barrandei

Obolus complexus Barr.
Obolus n. sp.

Lingulella (Baroisella) insons Barr.

Cystoidea - nejméně 4 druhy [at least 4 species]

jiná problematika [other problematic fossils]

Růžička (1931): rudní ložisko u Holoubkova (v Ouzkém) [ore deposit near Holoubkov (In Ouzký)]; Ouzký.

Euloma granulatum Ruž.

Eulomina n.g. mitratum Ruž.

Symphysurus Bröggeri Ruž.

Symphysurus bohemicus Klou.

Nileus Holoubkovensis Ruž.

Pliomera sp.

Lichas Kloučeki Ruž.

Cheirurus

Holometopus Grönwalli n. sp.

Olenus sp. III

pygidium

hypostomy [hypostoms]

Koliha (1937): Ouzký, près de Holoubkov [Ouzký near Holoubkov].

Obolus cf. kloučeki Kol.

Obolus (Lingulolobus) cf. feistmanteli (Barr.)

Obolus (Mickwitzella) cf. barrandei (Klou.)

Obolus compeexus Barr.

Lingulella insons (Barr.)

Micromitra (Iphidella) walcotti Růž.

Kutorgina Kolihai Růž.

Orbiculoidea sodalis undulosa (Barr.)

Siphonotreta krafti Růž.

Siphonotreta simulans Růž.

Acrotreta grandis Klou.

Billingsella incola (Barr.)

Billingsella grimmi (Barr.)

Orthis soror Barr.

Orthis potens Barr.

Clisospira sp.

Conularia sp.

Sphaeronites crateriformis Růž.

Sphaeronites batheri Růž.

Euloma granulatum Růž.

Eulomina mitratum Růž.

Apatocephalus sp.

Holubia bohemica Klou.

Olenus sp.

Niobe ferrigena Růž.

Nileus holoubkovensis Růž.

Symphysurus bohemicus Klou.

Megalaspis sp.

Cyrtometopus bohemicus Růž.

Lichas kloučeki Růž. 
Havlíček (1949a): Holoubkov.

Jivinella postcedens $\mathrm{n}$. sp.

Apheoorthina ferrigena $\mathrm{n}$. sp.

Apheoorthina bohemica n. sp.

Ocneorthis soror (Barrande, 1879)

Ocneorthis filia n. sp.

Orthambonites ruzickai n. sp.

Poramborthis lamelosa (Růžička, 1927)

Poramborthis grimmi (Barrande, 1879)

Poramborthis anomala n. sp.

Orthis? potens Barrande, 1879

Prantl \& Přibyl (1949a): "Mine Ouzký” near Holoubkov.

Hemibarrandia holoubkovensis (Růžička, 1926)

Havlíček (1951): Holoubkov.

Jivinella postcedens Havlíček, 1949

Apheoorthina bohemica Havlíček, 1949

Ocnerorthis soror (Barrande, 1879)

Ocnerorthis filia Havlíček, 1949

Orthambonites ruzickai Havlíček, 1949

Poramborthis anomala Havlíček, 1949

Vaněk (1959): Holoubkov, rudní ložisko v „Ouzkém" [Holoubkov, ore deposit in "Ouzký"]; býv. důl „Ouzký“ u Holoubkova [former mine "Ouzký” near Holoubkov].

Platylichas kloučeki (Růžička, 1926)

Prokop (1964): „V Ouzkém“ near Holoubkov.

Paleosphaeronires crateriformis (Růžička, 1927)

Vaněk (1965): „Na pískách“, ehemalige Grube „Ouzký" N Holoubkov ["Na Pískách”, former mine "Ouzký" north of Holoubkov]; Grube Ouzký bei Holoubkov [Ouzký Mine near Holoubkov]; Halden der ehemaligen Grube „Ouzký“ bei Holoubkov („Na pískách“) [mine dumps of the former "Ouzký” Mine near Holoubkov ("Na pískách”)]; „Ouzký“ bei Holoubkov ["Ouzký" near Holoubkov]; ehemalige Grube „Ouzký“ bei Holoubkov (“Na pískách”) [former "Ouzký" Mine near Holoubkov ("Na pískách")].

Holubaspis perneri (Růžička, 1926)

Hemibarrandia holoubkovensis (Růžička, 1926)

Pilekia bohemica (Růžička, 1926)

Eulomina mitrata (Růžička, 1926)

Eulomina granulata (Růžička, 1926)

Pharostomina oepiki Sdzuy, 1955

Platylichas klouceki (Růžička, 1926)

Přibyl \& Vaněk (1969): Holoubkov bei Rokycany [Holoubkov near Rokycany]; Holoubkov, „Ouzký“; Holoubkov („Ouzký“ bei Rokycany) [Holoubkov ("Ouzký" near Rokycany)].

Holoubkovia klouceki (Růžička, 1926)
Horný \& Bastl (1970): Ouzký near Holoubkov.

Pilekia bohemica (Ružička, 1926)

Diceratopyge? ferrigena (Ružicka, 1926)

Hemibarrandia holoubkovensis (Ružička, 1926)

Eulomina granulata (Ružička, 1926)

Platylichas klouceki (Ružička, 1926)

Eulomina mitrata (Ružička, 1926)

Pharostomina oepiki Sdzuy, 1955

Holubaspis perneri (Ružička, 1926)

Havlíček (1977): Holoubkov, abandoned ore mine "V Ouzkém".

Protambonites soror (Barrande, 1879)

Poramborthis grimmi (Barrande, 1879)

Poramborthis anomala Havlíček, 1949

Poramborthis cf. hispanica Havlíček, 1972

Apheorthina ferrigena Havlíček, 1949

Apheorthina bohemica Havlíček, 1949

Jivinella postcedens Havlíček, 1949

Jivinella ? ferrea Havlíček, 1977

Robertorthis holoubkovensis Havlíček, 1977

Robertorthis potens (Barrande, 1879)

Eoorthis ruzickai (Havlíček, 1949)

Ranorthis prima Havlíček, 1977

Havlíček (1980): Holoubkov.

Conotreta grandis (Klouček, 1915)

Mergl (1981): Holoubkov, abandoned iron ore mine "V Ouzkém"; Holoubkov, "V Ouzkém".

Orbithele discontinua sp. n.

Havlíček (1982a): Holoubkov, abandoned iron ore mine; Holoubkov; Holoubkov, iron ore mine; abandoned iron ore mine at Holoubkov.

Rosobolus robertinus $\mathrm{sp} . \mathrm{n}$.

Palaeoglossa bukovensis (Koliha, 1924)

Elkanisca sp. A

Broeggeria sp.

Lacunites walcotti (Růžička, 1927)

Kolihium kolihai (Růžička, 1927)

Eosiphonotreta krafti (Růžička, 1927)

Siphonobolus simulans (Růžička, 1927)

Ferrobolus catharinus sp. n.

Mergl (1984a): abandoned iron ore mine „Ouzký“ $\mathrm{N}$ of Holoubkov; Holoubkov, abandoned iron ore mine „Ouzký“.

Marcusodictyon exspenctans sp. $\mathrm{n}$.

Mergl (1984b): The mine "Ouzký" near Holoubkov; "Ouzký"

Hemibarrandia holoubkovensis (Růžička, 1926)

Holubaspis perneri (Růžička, 1926) 
Mergl (1994a): Holoubkov, abandoned mine "Ouzký"; Holoubkov.

Elkanisca obesa (Havlíček, 1980)

Mergl (1994b): Holoubkov

Ottenbyaspis (?) broeggeri (Růžička, 1926)

Hemibarrandia holoubkovensis (Růžička, 1926)

Holoubkovia klouceki (Růžička, 1926)

Anacheirurus bohemicus (Růžička, 1926)

Parapilekia ferrigena sp. $\mathrm{n}$.

Parabathycheilus sp.

Holoubkocheilus granulatus (Růžička, 1926)

Holubaspis perneri (Růžička, 1926)

Apatokephalus (?) sp.

Proteuloma cf. kettneri (Růžička, 1941)

Eulomina mitratum (Růžička, 1926)

Mergl (2002): Holoubkov (V Ouzkém).

Leptembolon insons (Barrande, 1879)

Hyperobolus feistmanteli (Barrande, 1879)

Thysanotos primus (Koliha, 1924)

Broeggeria ferraria sp. $\mathrm{n}$.

Rosobolus robertinus Havlíček, 1982

Elkanisca obesa (Havlíček, 1980)

Ferrobolus catharinus Havlíček, 1982

Orbithele discontinua Mergl, 1981

Acrotreta grandis Klouček, 1919

Eosiphonotreta krafti (Růžička, 1927)

Siphonobolus simulans (Růžička, 1927)

Celdobolus aff. mirandus (Barrande, 1879)

Kolihium kolihai (Růžička, 1927)

Lacunites walcotti (Růžička, 1927)

Petrocrania sp.

Mergl (2006): Holoubkov; Holoubkov, dumps of the abandoned "V Ouzkém" iron ore mine; Holoubkov ("V Ouzkém").

Ottenbyaspis broeggeri (Růžička, 1926)

Hemibarrandia holoubkovensis (Růžička, 1926)

Agerina ferrigena (Růžička, 1926)

Agerina clymene sp. n.

Holoubkovia klouceki (Růžička, 1926)

Anacheirurus bohemicus (Růžička, 1926)

Parapilekia ferrigena Mergl, 1994

Parabathycheilus krafti sp. n.

Holoubkocheilus granulatus (Růžička, 1926)

? dikelokephalinid

Holubaspis perneri (Růžička, 1926)

Apatokephalus aff. dagmarae Mergl, 1984

Platypeltoides sp.

Eulomina mitratum (Růžička, 1926)

Mergl \& Prokop (2006): Holoubkov, abandoned mine "V Ouzkém"; Holoubkov, abandoned iron ore mine called "V Ouzkém"; Holoubkov, "V Ouzkém".
Echinosphaerites concomitans Barrande, 1887 Glyptosphaerites ferrigena (Barrande, 1887)

Paleosphaeronites crateriformis (Růžička, 1927)

Pyrocystites sp.

Mergl (2009): Holoubkov; důl Ouzký u Holoubkova [Ouzký Mine near Holoubkov].

Marcusodictyon exspectans

Protambonites soror (Barrande)

Poramborthis grimmi (Barrande)

Poramborthis anomala Havlíček

Eoorthis ruzickai Havlíček

Robertorthis potens (Barrande)

Robertorthis holoubkovensis Havlíček

Kvania prima (Havlíček)

Apheoorthina ferrigena Havlíček

Glyptosphaerites ferrigena (Barrande)

Echinosphaerites concomitans (Barrande)

Poramborthis cf. grimmi (Havlíček)

Jivinella postcedens (Havlíček)

Apheoorthina bohemica Havlíček

Kvania sp.

Leptembolon insons (Barrande)

Rosobolus robertinus Havlíček

Rosobolus sp.

Broeggeria ferraria Mergl

Elkanisca obesa Havlíček

Ferrobolus catharinus Havlíček

Orbithele discontinua Mergl

Acrotreta grandis Klouček

Eosiphonotreta krafti (Růžička)

Siphonobolus simulans (Růžička)

Kolihium kolihai (Růžička)

Lacunites walcotti (Růžička)

Petrocrania sp.

Palaeosphaeronites crateriformis (Růžička)

Pyrocystites sp.

Mimospira sp.

Ottenbyaspis broeggeri (Růžička)

Hemibarrandia holoubkovensis (Růžička)

Agerina ferrigena (Růžička)

Anacheirurus bohemicus (Růžička)

Parapilekia ferrigena Mergl

Parabathycheilus krafti Mergl

Eulomina mitratum Růžička

Apatokephalus aff. dagmarae Mergl

Platypeltoides sp.

Holoubkocheilus granulatus (Růžička)

Holubaspis perneri (Růžička)

Holoubkovia klouceki (Růžička)

? Proteuloma sp. 

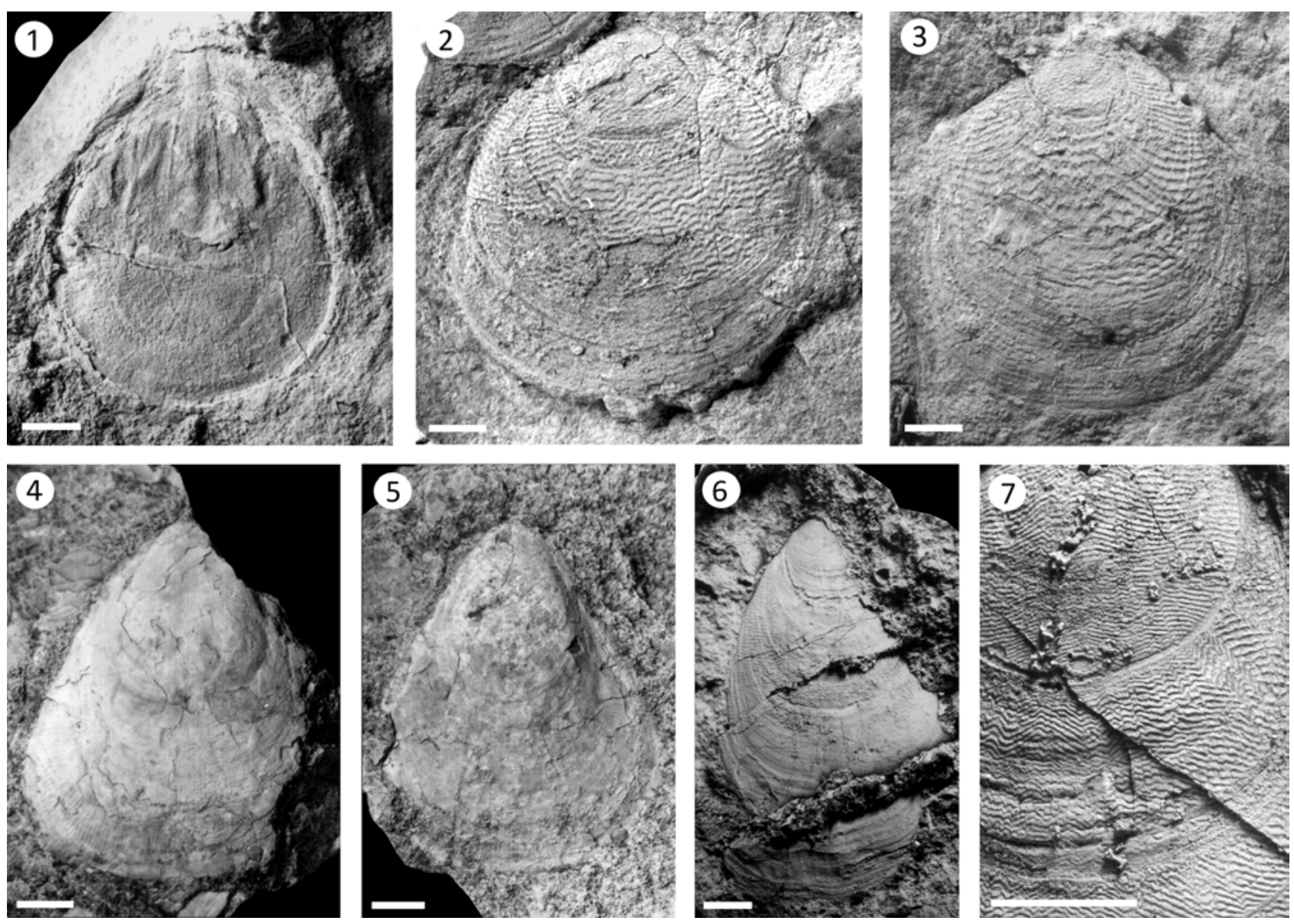

Plate I. Lingulate brachiopods with burrowing sculptures of the Třenice Formation from the Libečov - Na močidle locality. 1-3 - Westonisca lamellosa (Barrande, 1879), ventral valve MR 66901, dorsal valve MR 66899a, dorsal valve MR 66896b. 4, 5 - Libecoviella arachne (Barrande, 1879), dorsal valve MR 2186, dorsal valve NM L 32018. 6, 7 - Libecoviella ovata Havlíček, 1982, ventral valve, NM L 32028, detail of ornament, NM L 32027. Scale bars equal $2 \mathrm{~mm}$.

Plate II. Lingulate brachiopods of the Třenice Formation. Localities: Holoubkov - Ouzký (1-4, 6, 9, 12, 13, 17-25), Zbiroh Bukov (quarries) (5), Cheznovice - Žlebec (11), Krušná hora Hill (14-16).

1-4 - Acrotreta grandis Klouček, 1916, dorsal valve NM L 36747, ventral valve MN L 36749, ventral valve (two views) MR 21501. 5 - Teneobolus bukovensis (Havlíček, 1982), dorsal valve NM L 36715. 6 - Orbithele discontinua Mergl, 1981, ventral valve NM L 36737. 7 - Orbithele secedens (Barrande, 1879), ventral valve NM L 36742. 8 - Elkanisca obesa (Havlíček, 1980), dorsal valve MR 65773. 9 - Petrocrania sp., dorsal valve MR 23396. 10 - Expellobolus expulsus (Barrande, 1879), dorsal valve NM L 30790. 11 - Rosobolus magnus Mergl, 2002, ventral valve PCZCU 603b. 12, 13 - Rosobolus robertinus Havlíček, 1982, ventral valve MR 21833, dorsal valve MR 21467. 14-16 - Hyperobolus feistmanteli (Barrande, 1879), dorsal valve MR 21964, ventral valve MR 21938, dorsal valve MR 22780. 17-19 - Ferrobolus catharinus Havlíček, 1982, ventral valve MR 21837, ventral valve NM L 18125, dorsal valve MR 21835. 20 - Eosiphonotreta krafti (Růžička, 1927), dorsal valve MR 23744. 21 - Siphonobolus simulans (Růžička, 1927), ventral valve MR 21692. 22, 23 - Kolihium kolihai (Růžička, 1927), dorsal valve MR 21519, ventral valve, NM L 18129. 24, 25 - Lacunites walcotti (Růžička, 1927), ventral valve MR 22015, ventral valve NM L 36757. Scale bars equal $2 \mathrm{~mm}(1-13,17-25)$ and $10 \mathrm{~mm}(14-16)$.

Plate III. Orthid brachiopods of the Třenice Formation from the Holoubkov - Ouzký locality.

1-3 - Kvania prima (Havlíček, 1977), dorsal valve, MR 21470, ventral valve MR 21471, ventral valve MR 21472. 4-6 - Eoorthis ruzickai Havlíček, 1949, dorsal valve, MR 21724a, ventral valve MR 21724b, ventral valve MR 21462. 7-9 - Apheoorthina ferrigena Havlíček, 1949, ventral valve MR 21719, ventral valve MR 21720, dorsal valve MR 21717. 10-12 - Apheoorthina bohemica Havlíček, 1949, dorsal valve MR 21576, dorsal valve MR 21575, dorsal valve MR 21691. 13-15 - Jivinella postcedens Havlíček, 1949, dorsal valve MR 21814, ventral valve MR21699, ventral valve MR 21469. Scale bars equal 2 mm. 

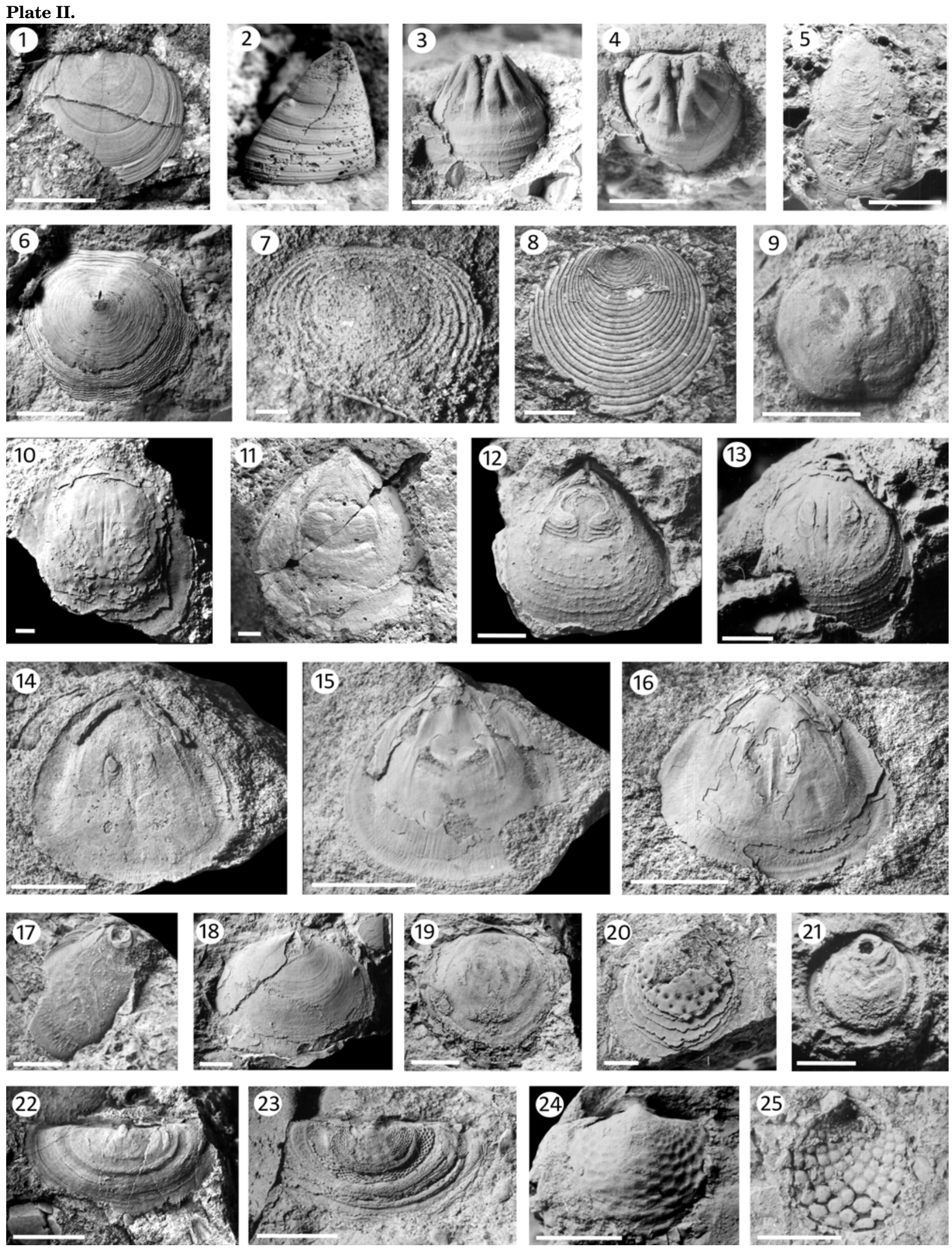


\section{Plate III.}
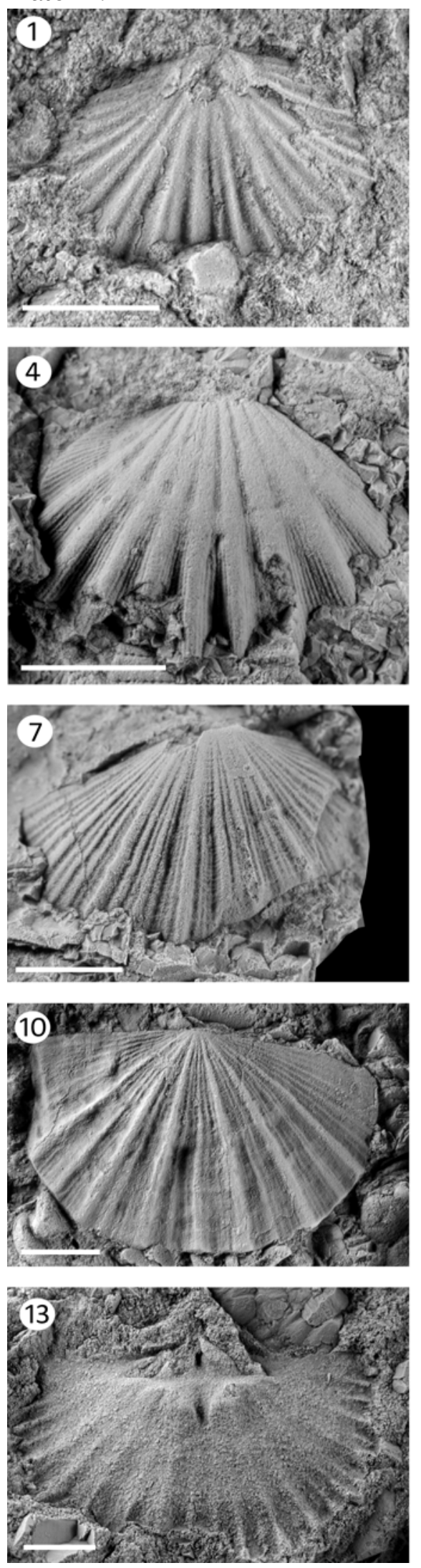
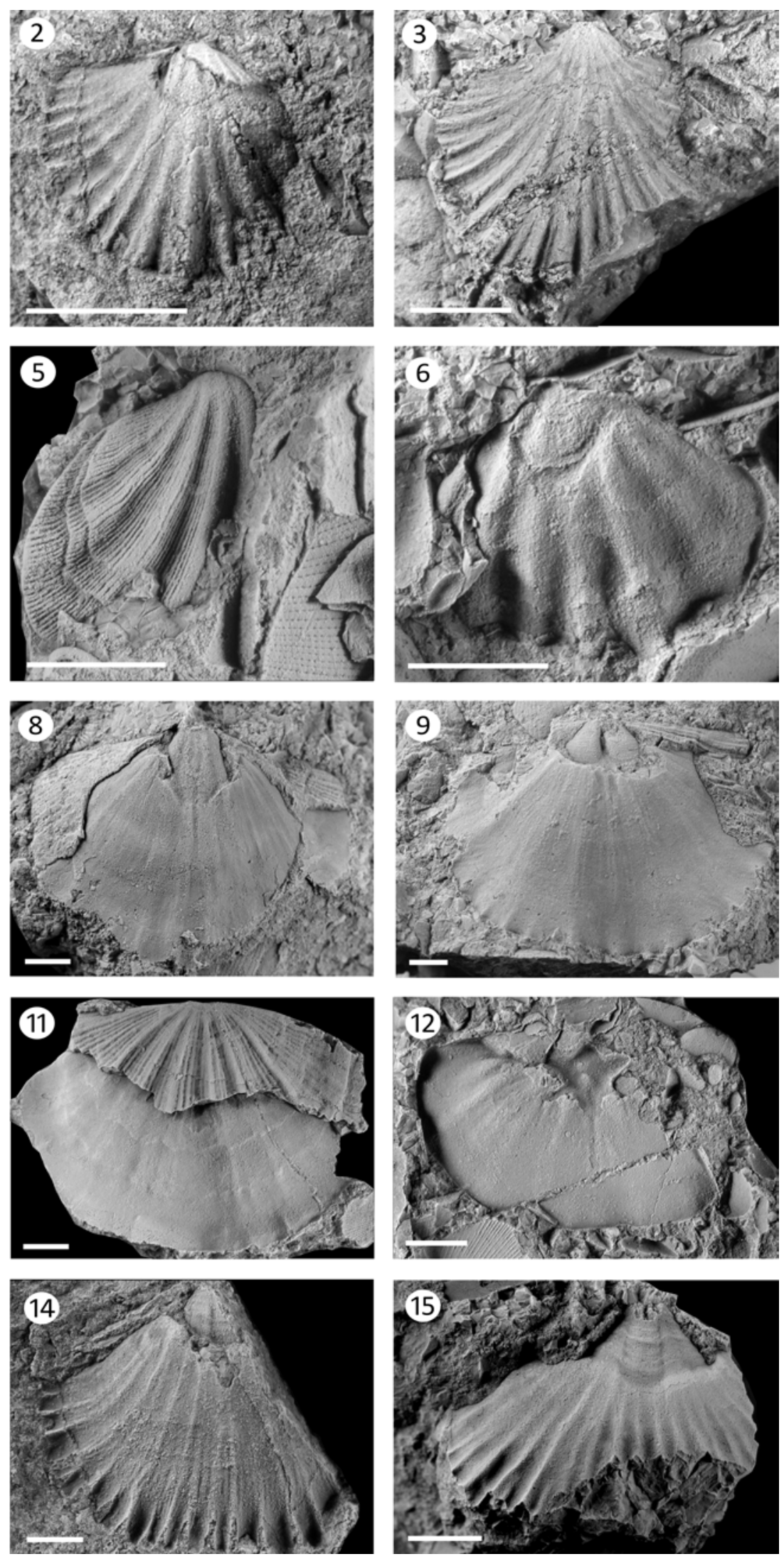
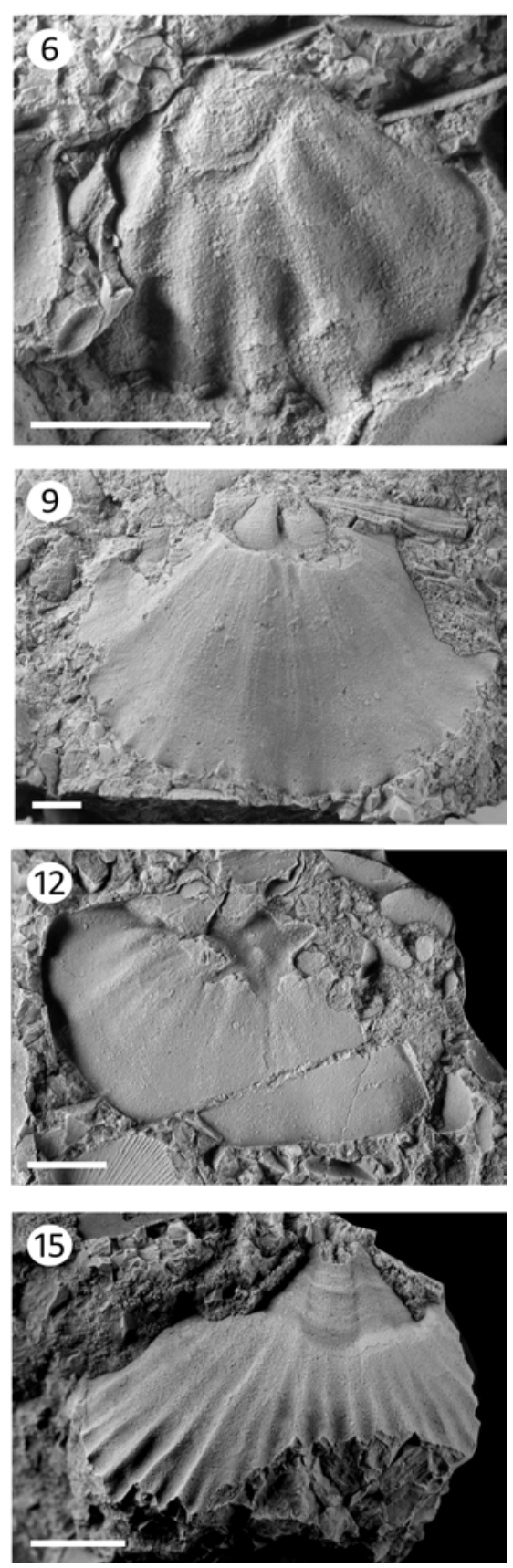

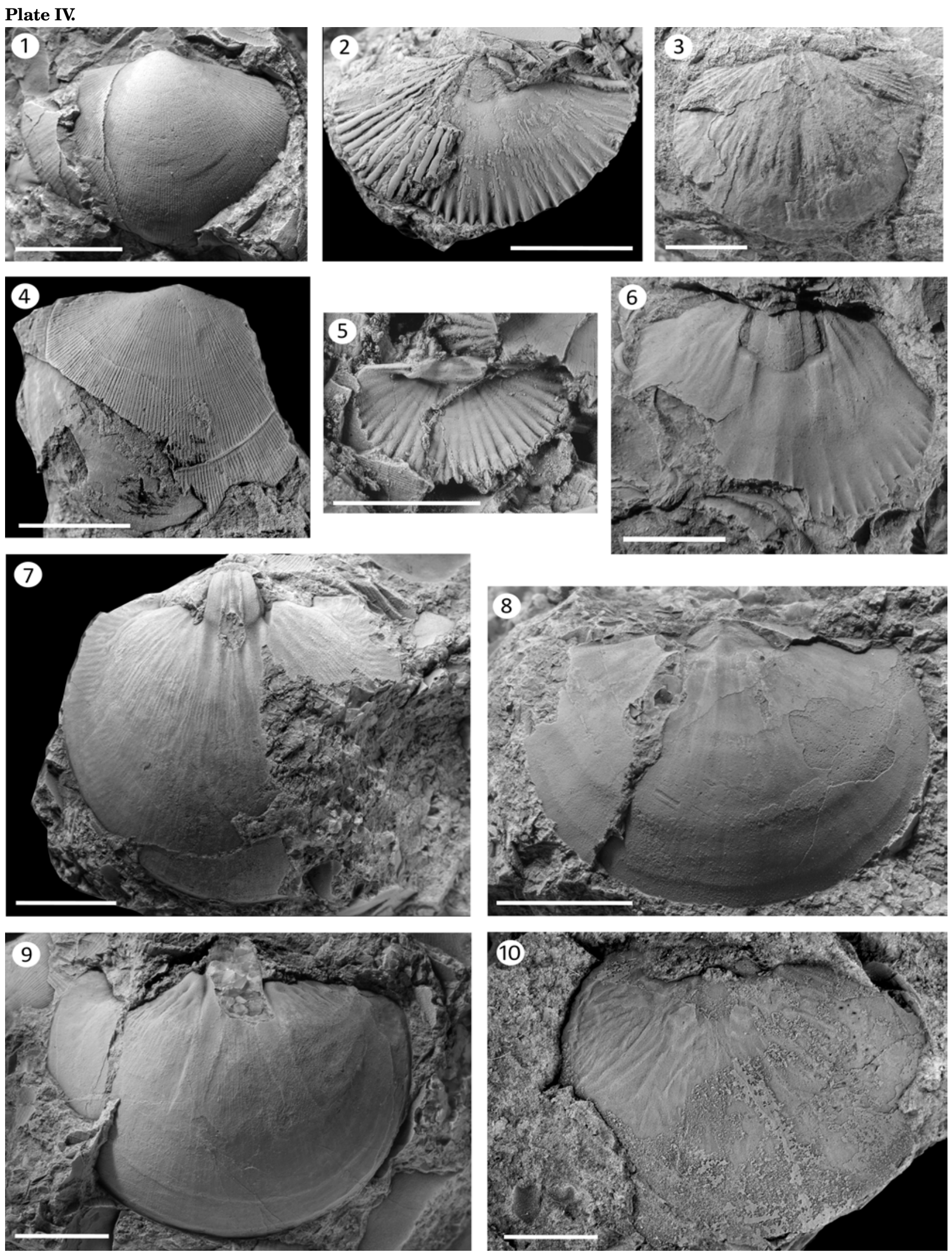
Plate V.
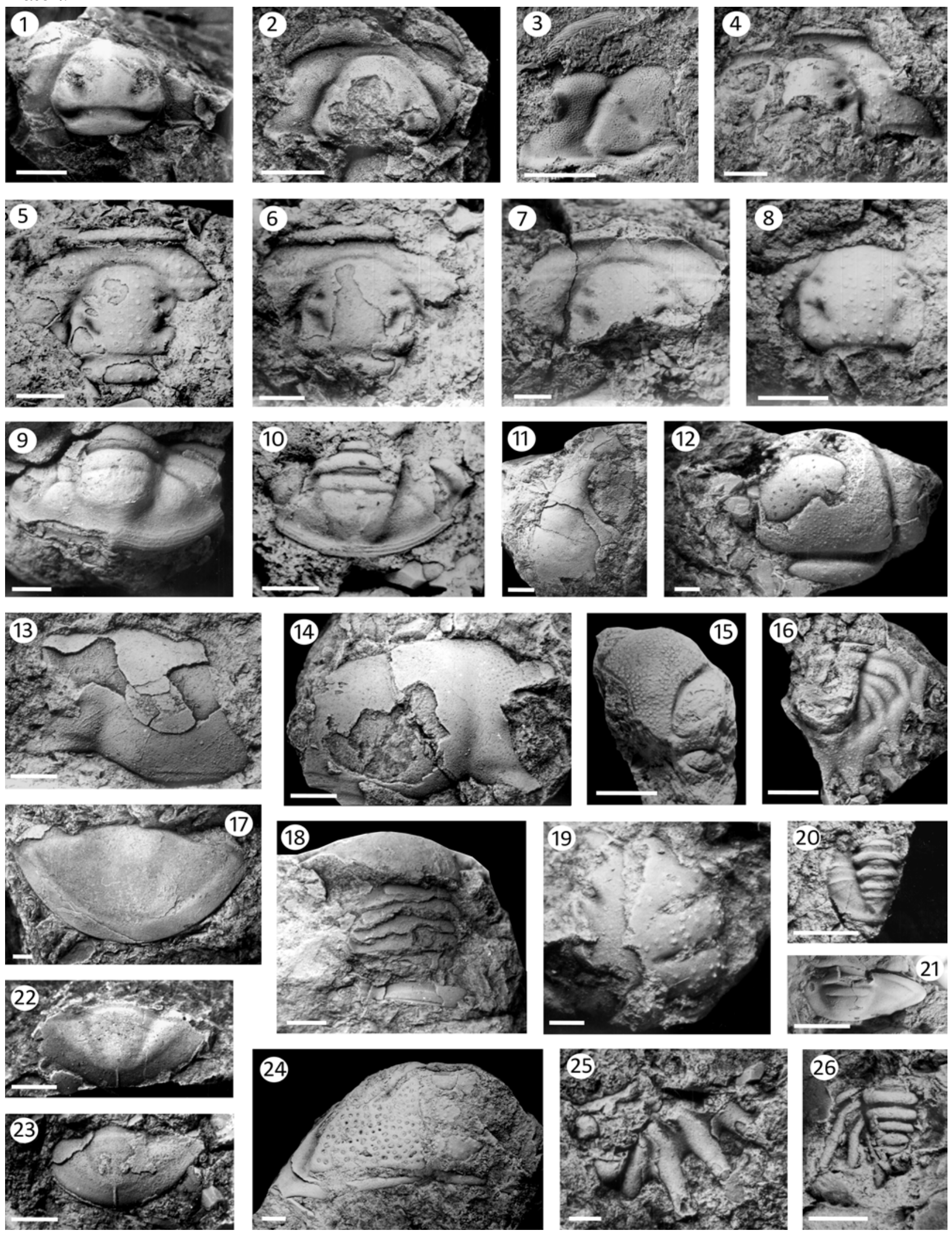


\section{Plate VI.}
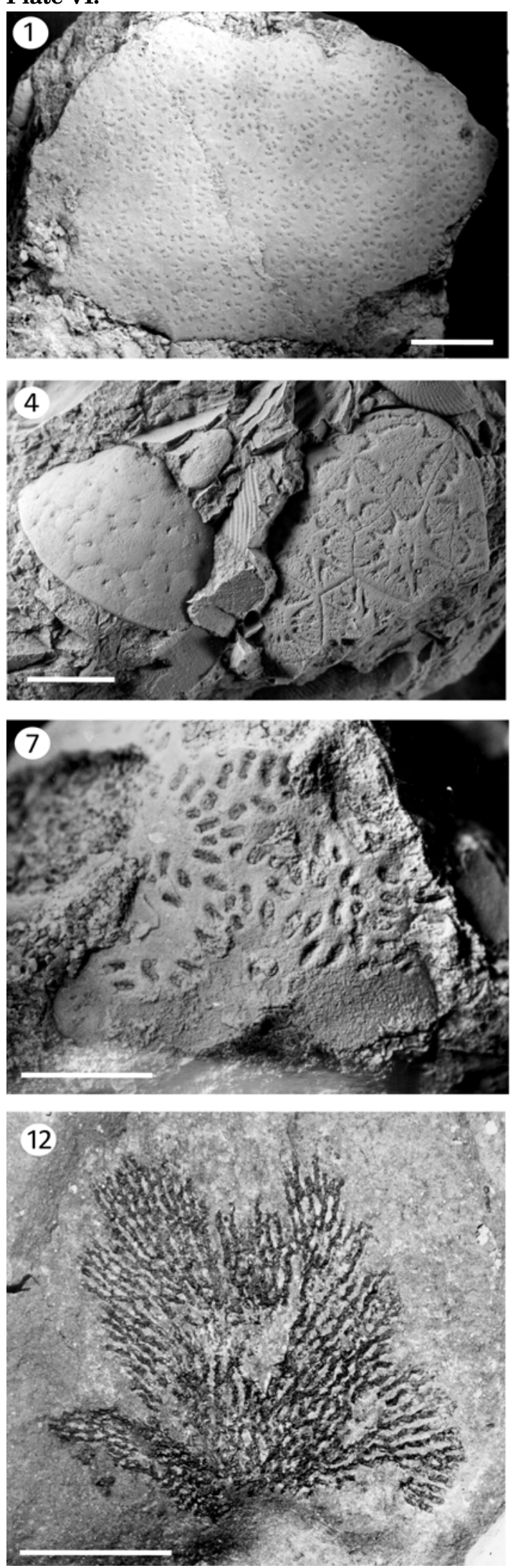
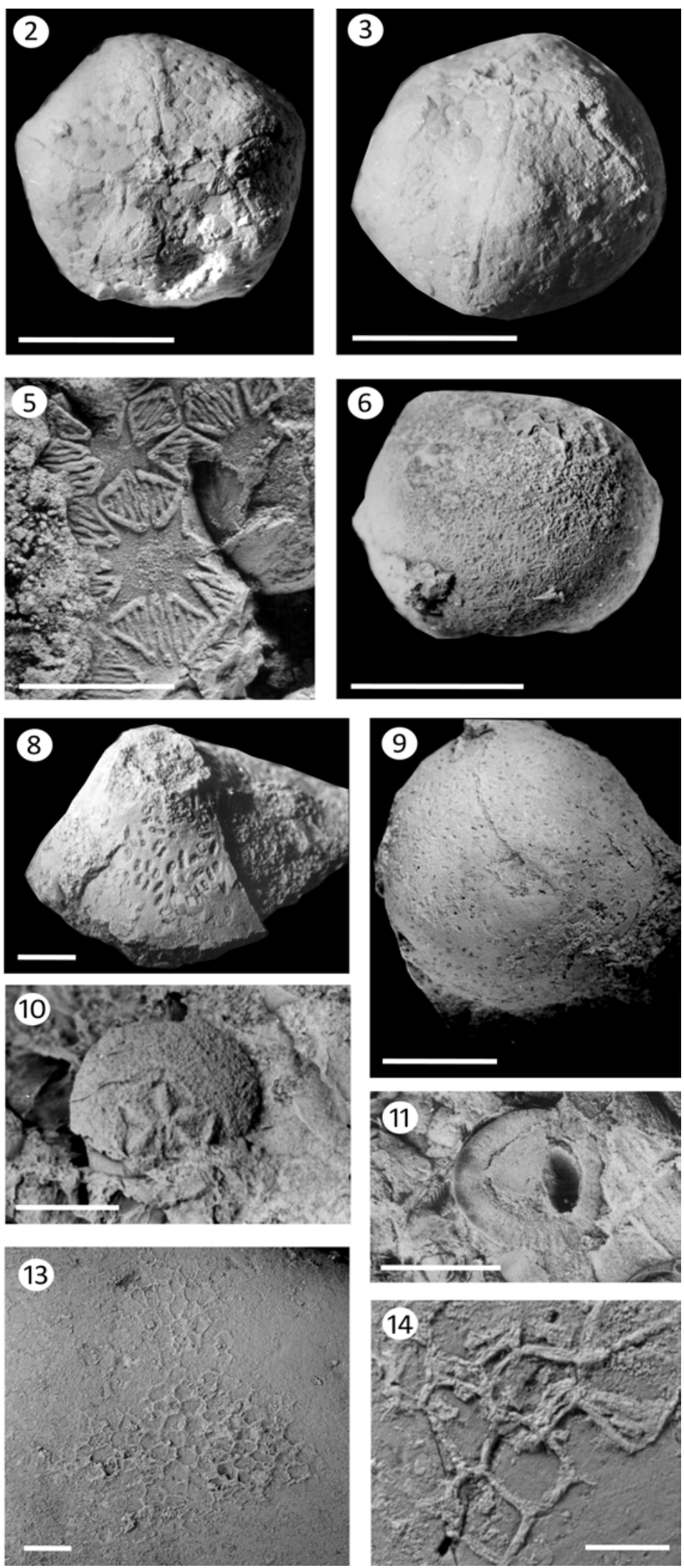
Mergl (2011): Holoubkov, abandoned mine „V Ouzkém".

Poramborhis grimmi (Barrande, 1879)

Updated list of fauna:

Cyathophycus? sp.

Marcusodictyon exspectans Mergl, 1984

Leptembolon insons (Barrande, 1879)

Hyperobolus feistmanteli (Barrande, 1879)

Thysanotos primus (Koliha, 1924)

Broeggeria ferraria Mergl, 2002

Rosobolus robertinus Havlíček, 1982

Elkanisca obesa (Havlíček, 1980)

Ferrobolus catharinus Havlíček, 1982

Orbithele discontinua Mergl, 1981

Acrotreta grandis Klouček, 1919

Eosiphonotreta krafti (Růžička, 1927)

Siphonobolus simulans (Růžička, 1927)

Kolihium kolihai (Růžička, 1927)

Lacunites walcotti (Růžička, 1927)

Petrocrania sp.

Protambonites soror (Barrande, 1879)

Poramborthis grimmi (Barrande, 1879)

Poramborthis anomala Havlíček, 1949

Poramborthis cf. hispanica Havlíček, 1972

Eoorthis ruzickai (Havlíček, 1949)

Jivinella postcedens Havlíček, 1949
Jivinella ? ferrea Havlíček, 1977

Robertorthis potens (Barrande, 1879)

Robertorthis holoubkovensis Havlíček, 1977

Kvania prima (Havlíček, 1977)

Apheoorthina ferrigena Havlíček, 1949

Apheoorthina bohemica Havlíček, 1949

Mimospira cf. helmhackeri (Perner, 1900)

Ottenbyaspis broeggeri (Růžička, 1926)

Hemibarrandia holoubkovensis (Růžička, 1926)

Agerina ferrigena (Růžička, 1926)

Agerina clymene Mergl, 2006

Holoubkovia klouceki (Růžička, 1926)

Anacheirurus bohemicus (Růžička, 1926)

Parapilekia ferrigena Mergl, 1994

Parabathycheilus krafti Mergl, 2006

Holoubkocheilus granulatus (Růžička, 1926)

? dikelokephalinid

Holubaspis perneri (Růžička, 1926)

Apatokephalus aff. dagmarae Mergl, 1984

Platypeltoides sp.

Eulomina mitratum (Růžička, 1926)

Proteuloma sp.

Echinosphaerites concomitans Barrande, 1887

Glyptosphaerites ferrigena (Barrande, 1887)

Paleosphaeronites crateriformis (Růžička, 1927)

Pyrocystites sp.

Plate IV. Rhynchonelliformean brachiopods of the Třenice Formation from the Holoubkov - Ouzký locality. 1, 4, 7, 9, 10 Poramborthis grimmi (Barrande, 1879), ventral valve MR 21685, dorsal valve MR 21727, ventral valve MR 21524a, ventral valve MR 21457, dorsal valve NM L 39311. 8 - Poramborthis anomala Havlíček, 1949, dorsal valve MR 21524b. 2,5 - Robertorthis holoubkovensis Havlíček, 1977, ventral valve MR 22574, dorsal valve MR 21690. 3, 5 - Protambonites soror (Barrande, 1879), dorsal valve MR 21476, ventral valve PCZCU 1899. Scale bars equal $5 \mathrm{~mm}$.

Plate V. Trilobites of the Třenice Formation from the Holoubkov - Ouzký locality. 1-3 Eulomina mitratum (Růžička, 1926), cranidium NM L 18886, cranidium NM L 18928, cranidium NM L 18928. 4-10 - Holoubkocheilus granulatus (Růžička, 1926), cranidium JV 2659, cranidium NM L 18884, cranidium NM L 30822, cranidium NM L 18918, cranidium NM L 30821, pygidium JV 2656, pygidium NM L 18885. 11 - Platypeltoides sp., librigena NM L 38507. 12 - Holubaspis perneri (Růžička, 1926), cranidium JV 2471. 13, 14, 17 - Hemibarrandia holoubkovensis (Růžička, 1926), cranidium NM L 18901, cranidium NM L 30824, pygidium NM L 18922. 15, 16 - Holoubkovia klouceki (Růžička, 1926), cranidium NM L 18909, pygidium NM L 11437. 18, 22, 23 - Ottenbyaspis? broeggeri (Růžička, 1926), cephalon with thorax NM L 18905, pygidium NM L 18921, pygidium NM L 30834a. 19, 24, 25 - Parapilekia ferrigena Mergl, 1994, cranidium NM L 18892, cranidium NM L 18907, pygidium NM L 18924. 20 - Agerina ferrigena (Růžička, 1926), pygidium NM L 18932. 21 - Proteuloma sp., pygidium MR 21451b. 26 - Anacheirurus bohemicus (Růžička, 1926), pygidium NM L 18908. Scale bars equal 2 mm.

Plate VI. Other fauna of the Třenice Formation from the Holoubkov - Ouzký (1-11, 13, 14), and Břežany - "Na Chrástnici" Quarry (12) localities. 1-3 - Glyptosphaerites ferrigena (Barrande, 1887), large theca NM L 37999, complete theca (two views), NM L 10618. 4 - fragments of thecae of Glyptosphaerites ferrigena (Barrande, 1887) (left) and Echinosphaerites concomitans Barrande, 1887 (right). 3-6 - Echinosphaerites concomitans Barrande, 1887, humatiorhombs NM L 38016, complete theca NM L 13111. 7-10 - Paleosphaeronites crateriformis (Růžička, 1927), detail of theca with peripores and the theca NM L 5847, theca NM L 38000, peristome NM L 16161. 11 - isolated columnalia NM L 38015. 12 - Dictyonema intermedium Prantl et Přibyl, 1949, flattened rhabdosome NM L 35552. 13, 14 - Marcusodictyon exspectans Mergl, 1984, zoarium encrusting a pebble and detail of zoarium, PCZCU 1897, PCZCU 1898. Scale bars equal $10 \mathrm{~mm}(1-3,6,9,12), 2 \mathrm{~mm}(4,5,7,8,10$, $11,13)$ and $1 \mathrm{~mm}(14)$. 

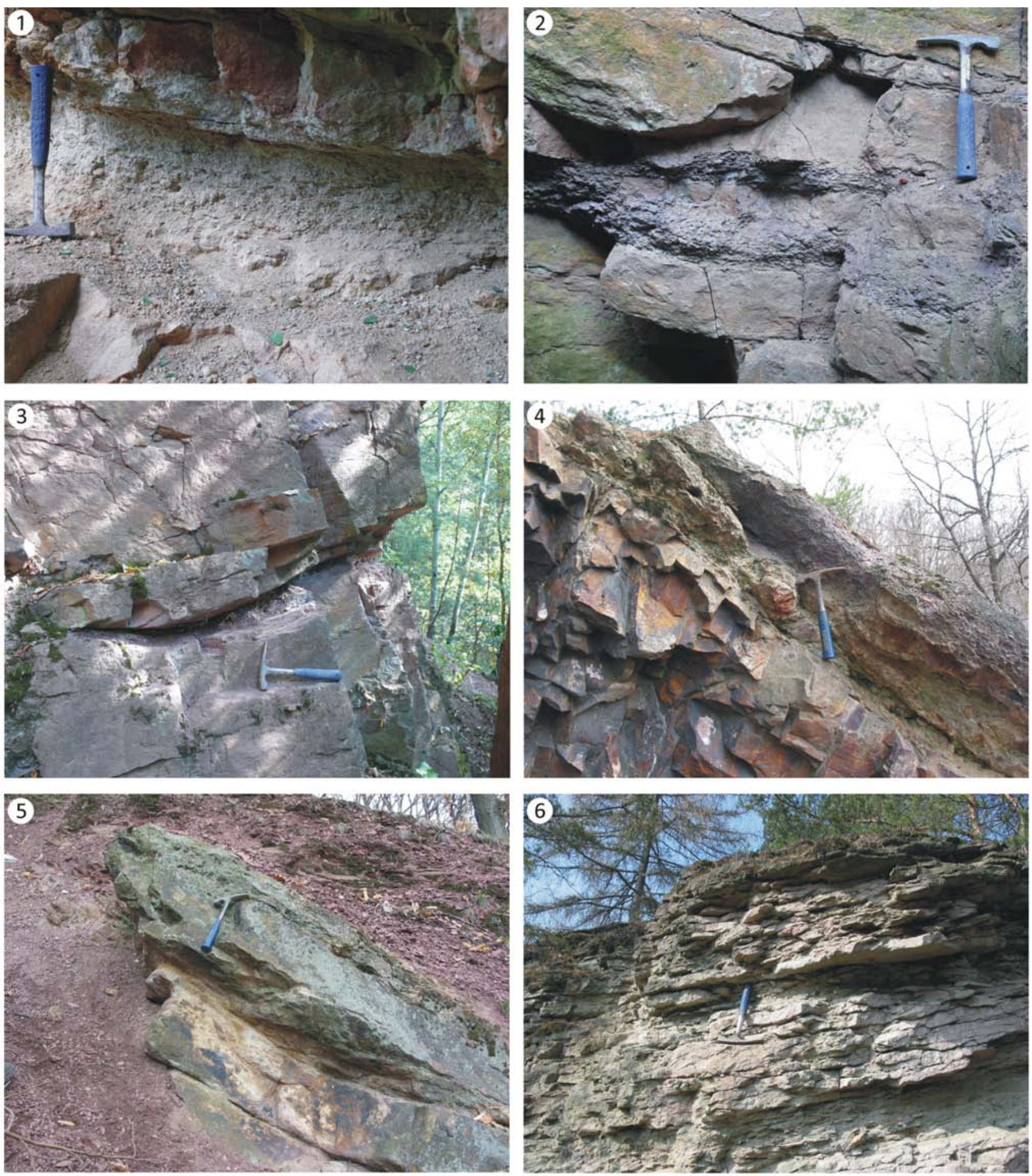

Plate VII. Main lithofacies exposed in the localities of the Třenice Formation. 1 - volcanigenic sandy conglomerate, old quarry at Cheznovice - Žlebec; 2 - Volcanigenic conglomerate enclosed within massive lithic sandstones, Kleštěnice - Jalový potok Brook; 3 - Cross-bedded middle-grained lithic sandstones, Jívina - quarries; 4 - Polymictic clast-supported conglomerate above the Cambrian fine to middle-grained quartz sandstone, basal part of the Třenice Formation, Medový Újezd; 5 - Lithic sandstones of the uppermost part of the Třenice Formation overlain by conglomerated layer (above top of the hammer) and reddish siltstones corresponding to the Klabava Formation, Medový Ujezd; 6 - Strongly weathered cross-bedded sandstones with layer of volcanigenic sandstones, Cerhovice - Cerhovská hora Hill. 


\section{Horní Kvaň - field}

Geography: Debris in the fields extended along a low ridge near the south margin of the village of Kvaň, some $900 \mathrm{~m}$ east of the monastery in Zaječov. Cadastre of Kvaň, District of Beroun. Mílina Formation also occurs at this locality.

Lithology: Lithic sandstone.

Mergl (2002): Kváň (field); Kváň (pole - field).

Acrotreta grandis Klouček, 1919

Updated list of fauna:

Acrotreta grandis Klouček, 1919

\section{Hostivice}

Geography: Exact location not known, probably from no longer discernable quarry west of the centre of the town of Hostivice.

Lithology: Sandstone.

Klouček (1931a, b): Hostivice.

Obolus Feistmanteli Barr. typus

Orthis Kettneri Klou.

Havlíček (1949a): Hostivice.

Poramborthis kettneri (Klouček, 1915 MS.) n. sp.

Havlíček (1951): Hostivice.

Jivinella praecedens (Prantl a Růžička, 1941)

Poramborthis kettneri (Klouček, 1915 MS), Havlíček, 1949

Havlíček (1977): Hostivice.

Poramborthis grimmi (Barrande, 1879)

Updated list of fauna:

Poramborthis grimmi (Barrande, 1879)

Jivinella praecedens (Prantl et Růžička, 1941)

\section{Jívina - quarries}

Geography: Abandoned quarries in a now wooded area along the road from the village of Jívina to the town of Komárov, some $800 \mathrm{~m}$ north-northeast of the centre of Jívina. Cadastre of Jívina, District of Beroun.

Lithology: Nine meters of succession exposed. Cross-bedded mid to coarse-grained greenish and brownish lithic sandstones predominate. Three meters above the base of exposed succesion is a 3 to 5 centimeters thick layer of coarse-grained volcanigenic sandstone, locally with current ripples. The uppermost part of the succesion fines upward into a mid to fine-grained lithic sandstone with subordinate thin (up to $2 \mathrm{~cm}$ thick) layers and lenses of siltstone and chert.
Remarks: The Třenice Formation outcrops in the eastern quarries from those situated along the road Jívina-Komárov. The western quarries have small outcrops of chert of the overlying Mílina Formation. The lower part of sandstone succession yielded a poor fauna, with rare Poramborthis grimmi. The upper part of the Třenice Formation has a distinct brownish layer, several $\mathrm{cm}$ thick, bearing abudant Jivinella praecedens, Thysanotos primus and chert lenses with minute, mostly undescribed acrotretaceans and siphonotretaceans. This fossiliferous level was nicely exposed in the western smaller quarry in the 1970's, but is now covered by slope debris.

? Klouček (1915): Komárov-Jívina.

Orthis Kettneri Kl. n. sp.

Obolella Feistmanteli Barr. sp.

Orthis sp. Barr.

Klouček (1919): Jívina; jižní lom na úpatí Jíviny u Komárova [southern quarry at the foot of the Jívina Hill near Komárov].

velká billingsella (orthis) radiálně rýhovaná [large radially ribbed Billingsella (Orthis)]

víčka acrotrety [opercula of Acrotreta]

Klouček (1920a): Jívina; sv. lomy na úpatí Jíviny u Komárova [north-western quarries at the foot of the Jívina Hill near Komárov].

Billingsella incola Barr. mut. praec.

Obolus cf. Barrandei Klou. n. sp.

Obolus n. sp.

Obolus Feistmanteli Barr.

Billingsella Kettneri Klou. n. sp.

Scolithus

Koliha (1924): Komárov-Jívina; Jívina.

Obolus (Lingulobolus) Feistmanteli (Barr.)

Obolus (Lingulobolus) Feistmanteli (Barr.) var. Barrandei prima n. var.

Kraft (1928): Jívina - Komárov; lomy u silnice z Jíviny do Komárova, veliký lom nejníže ke Komárovu [quarries near the road from Jívina to Komárov, the large quarry nearest to Komárov].

Obolus Feistmanteli

Lingulobolus Feistmanteli var. Barrandei

Orthis incola Barr.

Prantl \& Růžička (1941): Jívina.

Billingsella incola praecedens Klouček

Obolus (Mickwitzella) barrandei primus Koliha 
Havlíček (1949a): Jívina.

Jivinella praecedens (Prantl \& Růžička, 1941) Poramborthis kettneri (Klouček, 1915 MS.) n. sp. Tritoechia kodymi n. sp.

Havlíček (1951): Jívina.

Tritoechia kodymi Havlíček, 1949

Jivinella praecedens (Prantl et Růžička, 1941)

Poramborthis kettneri (Klouček, 1915 MS), Havlíček, 1949

Havlíček (1977): Quarry near the village of Jívina; Jívina.

Protambonites soror (Barrande, 1879)

Jivinella praecedens (Prantl and Růžička, 1941)

Poramborthis grimmi (Barrande, 1879)

Havlíček (1982a): Quarry east of Jívina; Jívina (quarry between Jívina and Komárov); Jívina.

Hyperobolus feistmanteli (Barrande, 1879)

Thysanotos primus (Koliha, 1924)

Mergl (1997b): Jivina (quarries along road JivinaKomárov); Jivina (east quarry).

Thysanotos primus (Koliha, 1924)

Mergl (2002): Jivina (old quarries); Jivina (staré lomy - old quarries).

Leptembolon insons (Barrande, 1879)

Thysanotos primus (Koliha, 1924)

Celdobolus aff. mirandus (Barrande, 1879)

Updated list of fauna:

Leptembolon insons (Barrande, 1879)

Thysanotos primus (Koliha, 1924)

Dactylotreta sp.

Celdobolus aff. mirandus (Barrande, 1879)

Protambonites soror (Barrande, 1879)

Poramborthis grimmi (Barrande, 1879)

Jivinella praecedens (Prantl et Růžička, 1941)

\section{Jívina Hill}

Geography: Natural exposures and slope debris on the east slope and the top of the Jívina Hill southwest and south of the village of Jívina. Cadastre of Jívina, District of Beroun.

Lithology: Greenish and brown-violet lithic sandstones.

Remarks: The exact site of fossiliferous layers is unknown, but near a small natural outcrops of the steep eastern slope of the hill. The remarkable fauna came from loose sandstone blocks in field east of the village of Jívina. The fossiliferous Míli- na Formation and the Olešná Member of the Klabava Formation also occur at this locality.

Havlíček (1977): south slope of Jívina Hill. Jivinella praecedens (Prantl and Růžička, 1941)

Mergl \& Prokop (2006): Jívina, slope debris; Jívina, slope debris near the Jívina-Komárov road. Paleosphaeronites crateriformis (Růžička, 1927)

Updated list of fauna:

Jivinella praecedens (Prantl et Růžička, 1941)

Paleosphaeronites crateriformis (Růžička, 1927)

\section{Kleštěnice - Jalový potok Brook}

Geography: Natural outcrops on the steep slope on the right bank (i.e. east the stream) of the Jalový potok Brook near the village of Kleštěnice, $1.3 \mathrm{~km}$ south-west of the castle in Komárov. Outcrops of the Trrenice Formation extend from the farm V Rochtě some $250 \mathrm{~m}$ up the stream along the brook. Cadastre of Kleštěnice, District of Beroun. Lithology: Fine to middle-grained massive and cross-bedded sandstones with lenticular intercalation of volcanigenic conglomerate. Cross-bedded sandstone contains decimeters thick beds of crossbed sets. Beds are separated by flat erosional surface. Massive sandstone forms beds with thickness from 30 centimeters to 1 meter and have erosional bases. Locally isolated pebbles of volcanic rocks occur above the bases of beds.

Volcanigenic conglomerate is generally matrix-supported, poorly sorted, with rhyolite subandular to subrounded pebbles up to $5 \mathrm{~cm}$ of diameter. Rarely the clasts of rhyolitic ignimbrite are also present (Hroch et al. 2012). Matrix is formed by clayey and sandy siltstone with angular fragments of volcanic glass. Conglomerate is without sedimentary structures and forms lenticular beds up to $35 \mathrm{~cm}$ thick and few meters to several tens of meters wide.

Remarks: Thick layers of the lithic sandstones outcrop in N part of the steep slope. Thick chert beds of the Mílina Formation and the Olešná Member of the Klabava Formation also occur at the locality. Fossils are very rare and poorly preserved.

Mergl (2002): Kleštěnice (section along the Jalový potok creek); Kleštěnice (profil podél Jalového potoka - section along the Jalový potok creek).

Thysanotos primus (Koliha, 1924)

Updated list of fauna:

Thysanotos primus (Koliha, 1924) 


\section{Krušná hora - Gabriela Mine}

Geography: Dumps of the abandoned iron ore Gabriela Mine located on the south-western slope of Krušná hora Hill near the village of Hudlice. Cadastre of Hudlice, District of Beroun.

Lithology: Greenish lithic sandstone.

Remarks: Large mine dump yielded abundant fossils of the Třenice Formation but fossils of the Klabava Formation can be sampled there, as well.

Mergl (2002): Krušná hora (Gabriela mine); Krušná hora (důl Gabriela - Gabriela mine).

Hyperobolus feistmanteli (Barrande, 1879)

Updated list of fauna:

Hyperobolus feistmanteli (Barrande, 1879)

\section{Krušná hora - gallery}

Geography: Dumps of abandoned mines on the eastern and northern slopes of Krušná hora Hill south of the village of Nový Jáchymov. Cadastre of Hudlice, District of Beroun.

Lithology: Greenish lithic sandstone.

Remarks: The locality is famous for nicely preserved large obolids, often with the original phosphatic shells. Judging from the stored material, fossils were common in particular layers. Fossils have been collected from small oucrops on the north slope of the Krušná Hora, but the exact location of outcrops from which they were obtained is unknown now. The best unweathered specimens, figured by Barrande (1879), were likely collected directly within the galleries of the mine when active in the mid of 19th century.

Koliha (1918): Krušná Hora (Dědičná štola) [Krušná Hora (Hereditary gallery)].

Obolus (Lingulobolus) Feistmanteli (Barr.) var. tenuilamellosa $\mathrm{n}$. var.

Koliha (1924): Františkova dědičná štola u Otročína [František's hereditary gallery near Otročín].

Obolus (Lingulobolus) Feistmanteli (Barr.)

Lingulella expulsa (Barr.)

Mergl (2002): Krušná hora (gallery).

Expellobolus expulsus (Barrande, 1879)

Hyperobolus feistmanteli (Barrande, 1879)

Orbithele secedens (Barrande, 1879)

Updated list of fauna:

Expellobolus expulsus (Barrande, 1879)

Hyperobolus feistmanteli (Barrande, 1879)

Orbithele secedens (Barrande, 1879)

\section{Krušná hora Hill}

Geography: Exact localities unknown on Krušná hora Hill. Cadastre of Hudlice, District of Beroun. Lithology: Lithic sandstone.

Remarks: There are numerous references to the Krušná hora locality without detailed locality data. The fossils likely derived from 19th century mine dumps. Unfortunately, old colletions often lack any details of the sampling sites.

Lipold (1863): Krušná hora.

Lingula Feistmantelli

Vála \& Helmhacker (1872): Krušná hora.

Lingula Feistmanteli Barr.

Vála \& Helmhacker (1874): Krušná hora

Lingula Feistmanteli Barr.

? Feistmantel (1878): Kruschna hora.

Discina

einen andern Obolus-artigen Brachiopoden [another species of brachiopod Obolus]

Barrande (1879): Kruschna Hora.

Lingula Feistmanteli. Barr.

Lingula expulsa. Barr.

Discina secedens. Barr.

Discina sodalis. Barr.

Katzer (1892): Krušná hora

Lingula Feistmanteli. Barr.

Jahn (1904a, 1904b): Krušná Hora

Obolella Feistmanteli

Koliha (1924): Krušná hora.

Obolus (Lingulobolus) Festmanteli (Barr.)

Lingulella expulsa (Barr.)

Mergl (1981): Krušná hora at Nový Jáchymov; Krušná hora.

Orbithele secedens (Barrande, 1879)

Havlíček (1982a): Krušná hora near Nový Jáchymov; Krušná hora.

Hyperobolus feistmanteli (Barrande, 1879)

Expellobolus expulsus (Barrande, 1879)

Orbithele secedens (Barrande, 1879)

Updated list of fauna:

Hyperobolus feistmanteli (Barrande, 1879)

Expellobolus expulsus (Barrande, 1879)

Orbithele secedens (Barrande, 1879) 


\section{Libečov - Na močidle}

Geography: Natural outcrops in the valley of the Chyňavský potok Brook some $600 \mathrm{~m}$ north-northeast the village of Libečov, $7.7 \mathrm{~km}$ north-north-east from the square in Beroun. Cadastre of Libečov, District of Beroun.

Lithology: Fine-grained sandstone.

Remarks: Fossils are abundant in fine-grained, laminated sandstones with accumulation pavements. Preservation is excellent, with original shell substance.

Vála \& Helmhacker (1872): močidlo východně od Libečova ["Močidlo" east of Libečov]; severovýchodně od Libečova na potoku Chyňavském na onom místě, kde zahýbá se [north-eastern of Libečov in the meander of the Chyňava Brook].

Lingula lamellosa Barr.

Vála \& Helmhacker (1874): Močidlo östlich von Libečov ["Močidlo" east of Libečov]; nordöstlich von Libečov am Chyňavabache, an jenem Orte, wo sich dieser biegt [north-eastern of Libečov in the meander of the Chyňava Brook].

Lingula lamellosa Barr.

Barrande (1879): Libetschov; Libetschow.

Lingula Arachne. Barr.

Lingula variolata. Barr.

Lingula lamellosa. Barr.

Lingula eximia. Barr.

Krejčí \& Helmhacker (1879): am Bache bei Libečov (Na močidle) [by brook near Libečov (Na močidle)]. Lingula lamellosa Barr.

Krejčí \& Helmhacker (1885): u potoka blíže Libečova [by brook near Libečov]; Močidlo (see Fig. 9 in the original paper).

Lingula lamellosa Barr.

Katzer (1892): Libečov

Lingula lamellosa Barr.

Jahn (1904a): „Na močidlech“ u Libečova ["Na močidlech" near Libečov]

Obolella (Lingula) Feistmanteli Barr. sp.

Obolella n. sp.

Lingula variolata Barr. nov. var.

Lingula expulsa Barr.

Lingula $\mathrm{n}$. sp.

Lingula $\mathrm{n}$. sp.

Lingula $\mathrm{n}$. sp.

Lingula $\mathrm{n}$. sp.
Discina sodalis Barr.

Discina n. sp.

Discina n. sp.

Acrothele secedens Barr. sp.

Lingula lamellosa Barr.

Lingula $\mathrm{n}$. sp.

Cf. Glotidia n. sp.

Jahn (1904b): „Na močidlech“ bei Libečov [“Na močidlech" near Libečov]

Lingula Arachne Barr.

Lingula eximia Barr.

Lingula variolata Barr.

Obolella (Lingula) Feistmanteli Barr. sp.

Obolella n. sp.

Lingula variolata Barr. nov. var.

Lingula expulsa Barr.

Lingula n. sp.

Lingula $\mathrm{n}$. sp.

Lingula $\mathrm{n}$. sp.

Lingula n. sp.

Discina sodalis Barr.

Discina n. sp.

Discina n. sp.

Acrothele (Discina) secedens Barr. sp.

Lingula lamellosa Barr.

Lingula $\mathrm{n}$. sp.

cf. Glotidia n. sp.

Woldřich (1916): Od Libečova na SSV, „Na močidle“ [NNE of Libečov, "Na močidle"]; (odkryvy) dole $\mathrm{u}$ potoka, nahoře u cesty a na svahu jižně od koty 361 [(outcrops) down by brook, up by road and on the slope south of elevation point 361].

Lingula lamellosa Barr.

Lingula obrysu trojúhelníkového, připomínající Obolella Feistmanteli Barr. [Lingula of the triangular outline resembling Obolella Feistmanteli Barr.]

zcela nový druh Linguly, dole široký, nahoře silně zúžený a protažený neuvedený ve velkém díle Barrande-ově [completely new species of Lingula, wide in its lower part, strongly narrowed in the upper part and elongated, not mentioned in the enormous work of Barrande]

Koliha (1918): Libečov.

Lingulella libečovensis $\mathrm{n}$. sp.

Klouček (1920a): pole na levém břehu potoka na Močidle [field on the left brook bank in Močidlo]. fragment nového obola [fragment of a new Obolus] 
eliptická miska menšího, snad rovněž nového lingulida [oval valve of a smaller, perhaps also new lingulid]

Koliha (1924): Libečov, lokalita zvaná „Na Močidle“ v údolí Chýňavského potoka [Libečov, locality called "Na Močidle" in the valley of the Chýňavský potok Brook]; „Na močidle“ u Libečova [“Na močidle" near Libečov].

Obolus (Westonia) lamellosus (Barr.)

Lingulella arachne (Barr.)

Heritsch (1928): Libečov.

Obolus lamellosus

Lingulella Arachne

? Koliha (1930a, 1930b): Libečov.

Obolus lamellosus (Barr.)

Havlíček (1982a): Libečov („Na močidle“); Libečov; Libečov (Na močidle).

Westonisca lamellosa (Bararnde, 1879)

Westonisca arachne (Barrande, 1879)

Mergl (1997a): Libečov („Na močidle“); „Na močidle“, Libečov.

Westonisca lamellosa (Barrande, 1879)

Libecoviella arachne (Barrande, 1879)

Mikuláš (2001): Libečov (stará lokalita na Močidlech u Chyňavského potoka) [Libečov (old locality na Močidlech near Chyňavský potok Brook)].

Planolites isp.

Phycodes isp.

Westonisca lamellosa (Barrande)

Poramborthis grimmi (Barrande)

Mergl (2002): Libečov (Na Močidle locality); Libečov (Na Močidle).

Libecoviella arachne (Barrande, 1879)

Westonisca lamellosa (Barrande, 1879)

Updated list of fauna:

Libecoviella arachne (Barrande, 1879)

Westonisca lamellosa (Barrande, 1879)

\section{Libečov - gallery}

Geography: Dump of the former gallery mine in the valley of Chyňavský potok Brook. Cadastre of Libečov, District of Beroun.

Lithology: Sandstone.

? Vála \& Helmhacker (1872): v obci Chyňavské blízko spojených cest, nejvýchodnější do Železné z Chyňavy vedoucí s cestou z Libečova k západu vedoucí a sice západně vedle cesty prvé; Libečovská štola kutní [in the village of Chyňava, near junction of roads, that of easternmost from Železná to Chyňava and that from Libečov to west, locality is west of the former road; Libečov gallery].

maličký Obolus sp. (posud nepopsaný) [minute Obolus sp. (undescribed yet)]

? Vála \& Helmhacker (1872): šachta číslo I ve Svárově [Mine No. I in Svárov].

Obolus sp.

Discina sp.

snad Syphonotreta sp. [perhaps Syphonotreta sp.]

? Vála \& Helmhacker (1872): šachta Svárovská III [Mine No. III in Svárov].

Obolus sp.

? Vála \& Helmhacker (1874): in der Gemeinde Chyňava in der nähe der vereinigten Wege, dem östlichen, welcher nach Železná und Chyňava fürth, mit dem aus Libečov gegen Westen führeden, und zwar westlich neben ersterem Wege; Libečover Schurfstollen [in the village Chyňava, near junction of roads, that of easternmost from Železná to Chyňava and that from Libečov to west, locality is west of the former road; Libečov gallery]. klein Obolus sp. (bis jetzt noch unbeschrieben) [minute Obolus sp. (undescribed yet)]

? Vála \& Helmhacker (1874): Schachte Nro. I in Svárov [Mine No. I in Svárov].

Obolus sp.

Discina sp. (ähnlich der Discina atenuata Barr.) [Discina sp. (similar to Discina atenuata Barr.)] vielleicht Syphonotreta sp. [perhaps Syphonotreta sp.]

? Vála \& Helmhacker (1874): Svárover Schachte Nro. III [Mine No. III in Svárov].

Obolus sp.

? Krejčí \& Helmhacker (1879): Svárov, Kamenná unweit Chýňava (W. Libečov) [Svárov and Kamenná near Chýňava (west of Libečov)].

Obolus

Discina

? Krejčí \& Helmhacker (1885): Svárov a Kamenná u Chýňavy (Z od Libečova) [Svárov and Kamenná near Chýňava (west of Libečov)].

Obolus

Discina 
Krejčí \& Feistmantel (1890): Svárov.

Lingula lamellosa

Woldřich (1916): rokle na Chrbině [a gully in Chrbina].

Lingula podobná Obolella Feistmanteli [Lingula similar to Obolella Feistmanteli]

? Woldřich (1916): Chrbina; štola Chrbinská [Chrbina Gallery].

četné zbytky ramenonožců [common fragments of brachiopods]

Klouček (1920a) štola Chrbinská [Chrbina Gallery]. Obolus lamellosus Barr.

Koliha (1924): Chrbina.

Obolus (Westonia) lamellosus (Barr.)

Mergl (1997a): Libečov (the dump of an Chrbina gallery).

Westonisca lamellosa (Barrande, 1879)

Mergl (2002): Chrbina (gallery); Chrbina (štola gallery).

Westonisca lamellosa (Barrande, 1879)

Updated list of fauna:

Westonisca lamellosa (Barrande, 1879)

\section{Medový Újezd}

Geography: Abandoned quarry on the south-western slope of the hill in the north-eastern of the centre of the village of Medový Újezd, above Medoújezdský potok Brook. Protected area of PP Medový Újezd. Cadastre of Medový Újezd, District of Rokycany.

Lithology: A 16 meters succession of the complete Třenice Formation. It is transgressive sitting on the Cambrian sandstone of the Ohrazenice Formation bounded by an unconformity. Its top is overlain by a $30 \mathrm{~cm}$ thick layer of sandy conglomerate corresponding to the base of the Klabava Formation.

A basal polymictic, clast-supported conglomerate of the Třenice Formation contains subrounded to rounded pebbles of rhyolite, quartz and the Neoproterozoic chert, matrix is formed by coarse to very coarse lithic sandstone. Cobbles are rare at the base of the unit. The basal conglomerate, $50-70 \mathrm{~cm}$ thick, fines upward into a cross-bedded lithic sandstone. This sandstone forms the main part of exposed succession. It is coarse-grained, the quartz grains and lithic fragments of volcanics and Neoproterozoic cherts and siltstones are the main components of clastic material. Volcanic glass fragments are locally present.

Remarks: The Třenice Formation is underlain by the poorly fossiliferous middle Cambrian and overlain by the Olešna Member of the Klabava Formation at this locality. Fossils are generaly rare and poorly preserved in coarse sandstone.

Lipold (1863): Medo-Aujesd. Lingula Feistmantelli Barr.

Koliha (1924): Medový Újezd u Holoubkova [Medový Újezd near Holoubkov].

Obolus (Lingulobolus) Feistmanteli (Barr.)

Kraft (1928): Medový Újezd; profil na severní straně vsi [section in the northern margin of the village].

Obolus Feistmanteli

Mergl (1997b): Medový Újezd (old quarry).

Thysanotos primus (Koliha, 1924)

Mergl (2002): Medový Újezd (quarry); Medový Újezd (lom - quarry).

Hyperobolus feistmanteli (Barrande, 1879)

Thysanotos primus (Koliha, 1924)

Updated list of fauna:

Hyperobolus feistmanteli (Barrande, 1879)

Thysanotos primus (Koliha, 1924

Orbithele secedens (Barrande, 1879)

\section{Medový Újezd - Hradiště}

Geography: Outcrops in an abandoned, small, shallow quarry on the hill south-eastern of the village. Cadastre of Medový Újezd, District of Rokycany. Lithology: Lithic sandstone.

Havlíček (1982a): Hradiště near Medový Újezd. Hyperobolus feistmanteli (Barrande, 1879)

Updated list of fauna:

Hyperobolus feistmanteli (Barrande, 1879)

\section{Popovice - Marešovka Quarry}

Geography: Small, abandoned, flooded quarry $880 \mathrm{~m}$ west-north-west of the crossing of roads in the centre of the village of Popovice, $3 \mathrm{~km}$ westsouth-west of the railway station in the town of Brandýs nad Labem, 17 km north-east of Prague's city centre. (Coordinates read from the map: N 50 $10^{\prime} 44^{\prime \prime}$, E $14^{\circ} 36^{\prime} 47^{\prime \prime}$.) Cadastre of Popovice u Brandýsa n. Lab., District of Praha-východ. 
Lithology: Coarse to fine, poorly bedded, sandstone.

Remarks: Well exposed sandstone with rare fossils.

Vaněk (1999): Abandoned quarry "Marešovka“. inarticulate brachiopods (not determinable)

Bavarilla cf. hofensis (Barrande)

Updated list of fauna:

Bavarilla cf. hofensis (Barrande, 1868)

\section{Skomelno - Na Solích}

Geography: Old mine dumps in the forest on the slope of $\mathrm{Na}$ Solích Hill $\sim 1.5 \mathrm{~km}$ east of the village of Skomelno. Cadastre of Vejvanov, District of Rokycany.

Lithology: Greenish volcanigenic clastics, some with haematitic cement.

Remarks: Fossils are frequent, with dominance of Acrotreta and small stromatolitic clasts.

Havlíček (1980): Skomelno (Na Solích).

Conotreta obesa sp. n.

Mergl (1994a): Skomelno, a dump of abandoned mine "Na Solích"; Skomelno.

Elkanisca obesa (Havlíček, 1980)

Mergl (2002): Skomelno (Na Solích).

Leptembolon insons (Barrande, 1879)

Elkanisca obesa (Havlíček, 1980)

Ferrobolus catharinus Havlíček, 1982

Orbithele discontinua Mergl, 1981

Acrotreta grandis Klouček, 1919

Updated list of fauna:

Leptembolon insons (Barrande, 1879)

Elkanisca obesa (Havlíček, 1980)

Ferrobolus catharinus Havlíček, 1982

Orbithele discontinua Mergl, 1981

Acrotreta grandis Klouček, 1919

\section{Svárov}

Geography: Exact locality unknown.

Katzer (1892): Svárov

Lingula lamellosa Barr.

Koliha (1924): Chrbina.

Obolus (Westonia) lamellosus (Barr.)

Updated list of fauna:

Westonisca lamellosa (Barrande, 1879)

\section{Těškov - Kněžský vrch Hill (quarry)}

Geography: Upper levels of the active quarry (now gone due to quarry activity) at Kněžský vrch Hill $1.2 \mathrm{~km}$ north-east of the chapel in the village of Těškov. Cadastres of Těškov and Lhota pod Radčem, District of Rokycany.

Lithology: Lithic sandstone with angular large clasts of rhyolite.

Remarks: Fossils are extremely fragmentary, forming the abundant bioclastic admixture in lithic sandstone. Collecting at this locality is not possible at the present time.

Mergl (2002): Těškov (Kněžský vrch Hill); Těškov (Kněžský vrch - Kněžský vrch Hill).

Elkanisca obesa (Havlíček, 1980)

Acrotreta grandis Klouček, 1919

Updated list of fauna:

Elkanisca obesa (Havlíček, 1980)

Acrotreta grandis Klouček, 1919

\section{Těškov - north-west of the village}

Geography: Isolated blocks of the rock north-west of the village of Těškov. Cadastre of Těškov, District of Rokycany.

Lithology: Coarse-grained lithic sandstone.

Remarks: Exact site is uknown.

Andrusov (1925): Menší výskyt u vsi Těžkova [Limited occurrence near the village of Těžkov].

Obolus Feistmanteli Barr.

Updated list of fauna:

Hyperobolus feistmanteli (Barrande, 1879)

\section{Týček}

Geography: Old, abandoned quarry "Na Čihadle" $\sim 1.3 \mathrm{~km}$ south-east of the village of Týček. Cadastre of Týček, District of Rokycany.

Lithology: Lithic sandstone.

Koliha (1924): Tejček u Zbiroha [Tejček near Zbiroh]; lom na Čihadle u Tejčku [a quarry at Čihadlo near Tejček].

Obolus (Lingulobolus) Feistmanteli (Barr.)

Kraft (1928): Čihadlo u Týčka [Čihadlo near Týček]. Obolus (Lingulobolus) Feistmanteli

Mergl (2002): Tejček.

Hyperobolus feistmanteli (Barrande, 1879)

Updated list of fauna:

Hyperobolus feistmanteli (Barrande, 1879) 


\section{Úvaly - shaft}

Geography: Shaft and related mine dumps along the western margin of the town of Úvaly. Cadastre of Úvaly u Prahy, District of Praha-východ.

Lithology: Lithic sandstone.

Remarks: All fossils were sampled in the first half of the 20th century. At present, the area is stronly urbanized and exact location of the sampled dumps cannot be determined. The fossils are poorly preserved, often tectonically deformed, as such, are lacking fine detail. The overlying Mílina Formation also occurs at this locality

Klouček (1920b): Ouvaly, 1 km západně od Ouval [1 km west of Úvaly].

Obolus feistmanteli Barr. typus 3 - 4 nové variety [Obolus feistmanteli Barr. typus and 3-4 new varieties]

Obolus f. Barrandei Klou. n. sp. [f. = probably cf.] Billingsella incola Barr. mut. praec. Klou.

Billingsella $\mathrm{n}$. sp. jemně brázděná a velká jako $B$. incola [Billingsella $\mathrm{n}$. sp. delicately wrinkled and of the same size as $B$. incola]

Billingsella n. sp. přes $3 \mathrm{~cm}$ dlouhá a 1,5 široká [Billingsella $\mathrm{n}$. sp. more than $3 \mathrm{~cm}$ long and $1.5 \mathrm{~cm}$ wide]

Billingsella n. sp. 1,5 cm dlouhá a 2,5 široká [Billingsella $\mathrm{n}$. sp. $1.5 \mathrm{~cm}$ long and $2.5 \mathrm{~cm}$ wide]

Billingsella $\mathrm{n}$. var. jako předešlá, ale dlouhá jako široká [as previous but as long as wide]

Lingulella n. sp.?

Orbiculoidea undulosa? Barr.

Acrotreta minima Barr.

Havlíček (1949a): Úvaly.

Jivinella praecedens (Prantl \& Růžička, 1941)

Nanorthis rara n. sp.

Tritoechia kolihai n. sp.

Tritoechia kodymi n. sp.

Tritoechia uvalica n. sp.

Havlíček (1950): Pokusná šachtice na okraji škvorecké obory [Test shaft in the margin of Śkvorec Game Preserve].

Thysanotos barrandei barrandei (Klouček)

Thysanotos barrandei primus (Koliha)

Lingulobolus feistmanteli minor Prantl a Růžička

Obolus giganteus Koliha

Tritoechia kolihai Havlíček

Tritoechia kodymi Havlíček

Tritoechia uvalica Havlíček
Nanorthis rara Havlíček

Jivinella praecedens (Prantl a Růžička)

neurčení zástupci rodu Lingulella Salter [undetermined members of genus Lingulella Salter] neurčení zástupci rodu Orbiculoidea D’Orbigny [undetermined members of genus Orbiculoidea D'Orbigny]

Havlíček (1977): Úvaly (reddish sandstone).

Protambonites soror (Barrande, 1879)

Nanorthis rara Havlíček, 1949

Havlíček (1982a): test-pit west of Úvaly; Úvaly (test pit), Úvaly.

Thysanobolus giganteus (Koliha, 1937)

Thysanobolus pirolus sp. $\mathrm{n}$.

Thysanotos primus (Koliha, 1924)

Vaněk (1999): Waste dump near the iron ore shaft west of Úvaly; locality No. 8.

Only references to older finds.

Mergl (2002): Úvaly (old dump); Úvaly (Stará halda - old dump).

Thysanotos primus (Koliha, 1924)

Updated list of fauna:

Thysanotos primus (Koliha, 1924)

Protambonites soror (Barrande, 1879)

Nanorthis rara Havlíček, 1949

\section{Úvaly}

Geography: Localities known only to be near the town of Úvaly. Cadastre of Úvaly u Prahy, District of Praha-východ.

Lithology: Sandstone.

Remarks: Mílina Formation also occurs at this site (first published by Klouček 1922a, 1922b). Updating the taxonomic list is not possible as there are no new data since the last study by Koliha in 1920's (references see below).

Krejčí \& Helmhacker (1879): zwischen dem Jägerhaus W. von Ouval und in Ouval selbst [between gamekeeper's lodge west of Ouvaly and Ouvaly itself].

Lingula lamellosa Barr.

Krejčí \& Helmhacker (1885): Ouvaly a Z od Ouval $\mathrm{u}$ Fidrholce [Ouvaly and west of Ouvaly near Fidrholec].

Lingula lamellosa Barr.

Koliha (1924): Ouvaly.

Obolus (Lingulobolus) Feistmanteli (Barr.) 
? Koliha (1926a, 1926b): Ouvaly.

Obolus cf. feistmanteli

orthidi

? Heritsch (1928): Ouvaly.

Obolus Feistmanteli

\section{Zbiroh - Bukov (old dump)}

Geography: Old mine dump on the north-western slope of the Bukov Hill $2.2 \mathrm{~km}$ south-west of the church at the town square in Zbiroh (N 49 50' 27.4" E $\left.13^{\circ} 44^{\prime} 49.9^{\prime \prime}\right)$. Cadastre of Zbiroh, District of Rokycany.

Lithology: Gradded conglomerate.

Remarks: Almost all fossils came from haematite cemented conglomerate sampled by C. Klouček in 1920 's. The only small fragment of a brachiopod shell was discovered later, in 1980's by one of us (M.M.), confirming the original samplig site. Only one species is present, often crushed, but the preservation of shell details is excellent.

Klouček (1924): Bukov; Bukov u Zbirohu [Bukov Hill near Zbiroh].

Obolus lamellosus Barr.

Lingulella Arachne (Lingulella libečovensis Koliha)

Andrusov (1925): Hromady u koty 550 na západ. svahu Bukova [Dumps near elevation point 550 on the western slope of the Bukov Hill].

Obolus lamellosus Barr.

Lingulella Arachne (libečovensis Koliha)

Kraft (1928): Bukov u Zbiroha [Bukov near Zbiroh]; dvě hromady u Josefského dolu [two dumps near the Josef Mine].

Obolus (Westonia) lamellosus Barr.

Lingulella Arachne Barr.

Havlíček (1982a): South-eastern foot of Bukov Hill near Zbiroh.

Westonisca ovata $\mathrm{sp} . \mathrm{n}$.

Mergl (1997a): Northern slope of Bukov Hill near Zbiroh, the dump of an old abandoned test pit; old test pit in N slope of Bukov Hill near Zbiroh.

Libecoviella ovata (Havlíček, 1982)

Mergl (2002): Zbiroh (Bukov, old dump); Zbiroh (Bukov, stará halda - Bukov, old dump).

Libecoviella ovata (Havlíček, 1982)

Updated list of fauna:

Libecoviella ovata (Havlíček, 1982)

\section{Zbiroh - Bukov (quarries)}

Geography: Two areas; the abandoned quarries on the southern slope of the Bukov Hill $\sim 2.4 \mathrm{~km}$ south-west of the church at the town square in Zbiroh and the abandoned quarry at the northnorth-eastern foot of Bukov Hill near the path to Švabín. Cadastre of Zbiroh, District of Rokycany. Lithology: Lithic sandstone lying on conglomerate. Remarks: Several quarries of variable size are present on south and south-east slope of the $\mathrm{Bu}$ kov Hill, some partially to completely filled by garbage and debris. Quarries are also wooded over, thus, the exact sampling sites are not known. The locality with a well preserved fauna in fine-grained haematitic sandstone is likely from the most eastward quarry.

Lipold (1863): Bukow, nach Südost getriebene Josephistollen [Bukow, the Josef Gallery digged south-eastward].

Lingula Feistmantelli Barr.

Klouček (1917): opuštěný menší lom na s.s.v. úpatí Bukova [abandoned small quarry at the northnorth-eastern foot of the Bukov Hill].

var. druhu Obolus? minimus Barr.? [variety of the species Obolus? minimus Barr.?]

několik nových druhů brachiopodů, mezi nimiž jsou obolelly, acrtotreta, acrothele (?) a snad i barroiselly [several new species of brachiopods, Obolella, Acrotreta, Acrothele (?) and perhaps Barroisella are among them]

Obolella Feistmanteli Barr.

Lingula (?) expulsa Barr.

Discina undulosa Barr.

Klouček (1919): Bukov.

Obolus Feistmanteli Barr.

Lingulella? expulsa Barr.

Lingulella? n. sp. I

Orbiculoidea sodalis Barr.

Orbiculoidea undulosa Barr.

Acrotreta minima var. grandis Klou. n. sp.

Acrotreta minima Barr.

Lingulella $\mathrm{n}$. sp. II

Klouček (1920a): sv. malý lom na úpatí Bukova $\mathrm{u}$ Zbirohu [small quarry at the north-eastern foot of the Bukov Hill near Zbiroh].

Ob. Feistmanteli

Orb. sodalis

Lingul. expulsa 
Koliha (1924): Bukov; Bukov u Zbirohu.

Obolus (Lingulobolus) Feistmanteli (Barr.)

Lingulella Bukovensis n. sp.

Lingulella expulsa (Barr.)

Orbiculoidea sodalis

Acrotreta sp.

Andrusov (1925): Kota 491 na vých. úpatí Bukova [Elevation point 491 at the eastern foot of Bukov Hill].

Obolus Feistmanteli Barr.

Lingulella expulsa Barr.

Lingulella $\mathrm{n}$. sp. I

Lingulella $\mathrm{n}$. sp. II

Orbiculoidea sodalis Barr, sp.

Orbiculoidea undulosa Barr, sp.

Acrotreta minima Barr.

Acrotreta minima var. grandis Klou.

Orthis (Billingsella) incola var. praec. Klou.

Kraft (1928): Bukov u Zbiroha [Bukov near Zbiroh]; lom při cestě vpravo od myslivny na jižním svahu Bukova [quarry near the road, right of the gamekeeper's lodge on the southern slope of the Bukov Hill].

\section{Obolus Feistmanteli}

Kraft (1928): Bukov u Zbiroha [Bukov near Zbiroh]. Obolus Feistmanteli

Orthis incola

Discina sodalis - velká i malá varieta [Discina sodalis - large as well as small varieties]

Acrotreta minima

Acrotreta minima var. grandis

Havlíček (1980): Bukov Hill near Zbiroh.

Conotreta grandis (Klouček, 1915)

Havlíček (1982a): Bukov near Zbiroh; south-eastern foot of Bukov Hill near Zbiroh, Bukov Hill near Zbiroh.

Rosobolus robertinus Havlíček, 1982

Thysanobolus pirolus sp. n.

Palaeoglossa bukovensis (Koliha, 1924)

Mergl (2002): Zbiroh (Bukov, old quarries); Zbiroh (staré lomy - old quarries).

Expellobolus expulsus (Barrande, 1879)

Teneobolus bukovensis (Koliha, 1924)

Hyperobolus feistmanteli (Barrande, 1879)

Orbithele secedens (Barrande, 1879)

Acrotreta grandis Klouček, 1919
Updated list of fauna:

Expellobolus expulsus (Barrande, 1879)

Teneobolus bukovensis (Koliha, 1924)

Hyperobolus feistmanteli (Barrande, 1879)

Acrotreta grandis Klouček, 1919

Orbithele secedens (Barrande, 1879)

\section{Updated list of fauna of the Třenice Formation}

Porifera

Cyathophycus? sp.

? Bryozoa

Marcusodictyon exspenctans Mergl, 1984

Brachiopoda

Expellobolus expulsus (Barrande, 1879)

Leptembolon insons (Barrande, 1879)

Libecoviella arachne (Barrande, 1879)

Libecoviella ovata (Havlíček, 1982)

Teneobolus bukovensis (Koliha, 1924)

Westonisca lamellosa (Barrande, 1879)

Hyperobolus feistmanteli (Barrande, 1879)

Thysanotos primus (Koliha, 1924)

Broeggeria ferraria Mergl, 2002

Rosobolus magnus Mergl, 2002

Rosobolus robertinus Havlíček, 1982

Elkanisca obesa (Havlíček, 1980)

Ferrobolus catharinus Havlíček, 1982

Orbithele discontinua Mergl, 1981

Orbithele secedens (Barrande, 1879)

Acrotreta grandis Klouček, 1919

Dactylotreta sp.

Celdobolus aff. mirandus (Barrande, 1879)

Eosiphonotreta krafti (Růžička, 1927)

Siphonobolus simulans (Růžička, 1927)

Kolihium kolihai (Růžička, 1927)

Lacunites walcotti (Růžička, 1927)

Petrocrania sp.

Protambonites kolihai (Havlíček, 1949)

Protambonites soror (Barrande, 1879)

Poramborthis grimmi (Barrande, 1879)

Poramborthis anomala Havlíček, 1949

Poramborthis cf. hispanica Havlíček, 1972

Eoorthis ruzickai (Havlíček, 1949)

Eoorthis? sp.

Jivinella praecedens (Prantl et Růžička, 1941)

Jivinella postcedens Havlíček, 1949

Jivinella ? ferrea Havlíček, 1977

Robertorthis holoubkovensis Havlíček, 1977 
Robertorthis potens (Barrande, 1879)

Kvania prima (Havlíček, 1977)

Apheoorthina ferrigena Havlíček, 1949

Apheoorthina bohemica Havlíček, 1949

Nanorthis rara Havlíček, 1949

Gastropoda

Mimospira cf. helmhackeri (Perner, 1900)

Trilobita

Ottenbyaspis broeggeri (Růžička, 1926)

Hemibarrandia holoubkovensis (Růžička, 1926)

Agerina ferrigena (Růžička, 1926)

Agerina clymene Mergl, 2006

Holoubkovia klouceki (Růžička, 1926)

Anacheirurus bohemicus (Růžička, 1926)

Parapilekia ferrigena Mergl, 1994

Parabathycheilus krafti Mergl, 2006

Holoubkocheilus granulatus (Růžička, 1926)

Bavarilla cf. hofensis (Barrande, 1868)

dikelokephalinid

Holubaspis perneri (R̊žička, 1926)

Apatokephalus aff. dagmarae Mergl, 1984

Platypeltoides sp.

Eulomina mitratum (Růžička, 1926)

Proteuloma sp.

Echinodermata

Echinosphaerites concomitans Barrande, 1887

Glyptosphaerites ferrigena (Barrande, 1887)

Paleosphaeronites crateriformis (Růžička, 1927)

Pyrocystites sp.

Graptolithina

Dictyonema intermedium Prantl et Přibyl, 1949

Callograptus kodymi Prantl et Přibyl, 1949

\section{ACKNOWLEDGEMENTS}

We thank anonymous reviewer for the valuble comments which improved our manuscript. This research was funded by project of Ministry of Culture No. RK01P03OMG022: Registr paleontologických lokalit ordoviku pražské pánve [Register of paleontological localities in the Ordovician of the Prague Basin]. Subsequently, it was finished and completed for publishing under the project of the West Bohemian Museum in Plzeň No. UUP 2012/05. Parts of the study was supported by Charles University in Prague through the projects PRVOUK P44 (to P. K.) and SVV261203 (to T. H.).

\section{REFERENCES}

Andrusov, D. 1925. Geologické poměry Zbirožska. Sborník Státního geologického ústavu československé republiky 5, 53-110.

Barrande, J. 1879. Systême silurien du centre de la Bohême. $1^{\text {èr }}$ Partie: Recherches Paléontologiques. Vol. 5. Classe des Mollusques. Ordre des Brachiopodes. Trois chapitres de texte et Planches. 226 pp., pls. 1-71. Prague and Paris.

Barrande, J. 1879. Systême silurien du centre de la Bohême. $1^{\text {èr }}$ Partie: Recherches Paléontologiques. Vol. 5. Classe des Mollusques. Ordre des Brachiopodes. Planches 72-153. Pls. 72-153. Prague and Paris.

Feistmantel, K. 1878. Über die Lagerungverhältnisse der Eisensteine in der Unterabtheilung $D_{1}$ des böhmischen Silurgebirges. Selbstverlag, Dr. Ed. Grégr, 15 pp.

Havlíček, V. 1949a. Orthoidea a Clitambonoidea z českého tremadoku. Sborník Státního geologického ústavu Československé republiky 16, 93144.

Havlíček, V. 1949b. Předběžná zpráva o geologických poměrech na Úvalsku. Věstník Státního geologického ústavu Československé republiky 14, 94-96.

Havlíček, V. 1950. Geologie úvalského staršího paleozoika. Sborník Státního geologického ústavu Československé republiky, Oddíl geologický 17, 141-184.

Havlíček, V. 1951. Ramenonožci českého ordoviku. Rozpravy Ústředního ústavu geologického 13 (for 1950), 1-133.

Havlíček, V. 1977. Brachiopods of the order Orthida in Czechoslovakia. Rozpravy Ústředního ústavu geologického 44, 1-327.

Havlíček, V. 1980. Conotreta Walcott (Brachiopoda) in the Lower Ordovician of Bohemia. Věstník Ústředního ústavu geologického 55(5), 297-299.

Havlíček, V. 1981. Development of a linear sedimentary depression exemplified by the Prague Basin (Ordovician-Middle Devonian; Barrandian area - Central Bohemia). Sborník geologických věd, Geologie 35, 7-48.

Havlíček, V. 1982a. Lingulacea, Paterinacea, and Siphonotretacea (Brachiopoda) in the Lower Ordovician sequence of Bohemia. Sborník geologických věd, Paleontologie 25, 9-82.

Havlíček, V. 1982b. Ordovician in Bohemia: Development of the Prague Basin and its benthic com- 
munities. Sborník geologických věd, Geologie 37, 103-136.

Havlíček, V. 1998. Ordovician, 41-79. In Chlupáč, I., Havlíček, V., Kříž, J., Kukal, Z. \& Štorch, P. Palaeozoic of the Barrandian (Cambrian to Devonian). Czech Geological Survey, Prague.

Havlíček, V. \& Fatka, O. 1992. Ordovician of the Prague Basin (Barrandian area, Czechoslovakia, 461-471. In Webby, B.D. \& Laurie, J.R. (eds) Global Perspectives on Ordovician Geology. Balkema, Rotterdam.

Havlíček, V. \& Vaněk, J. 1966. The Biostratigraphy of the Ordovician of Bohemia. Sbornik geologických věd, Řada Paleontologie 8, 7-69.

Heritsch, F. 1928. Das Silur von Böhmen. Geologische Rundschau 19 (4), 321-344.

Horný, R. \& Bastl, F. 1970. Type Specimens of Fossils in the National Museum Prague, Volume 1, Trilobita. Museum of Natural History, Prague, 354 pp. Prague.

Hroch, T., Rajchl, M., Kraft, P. \& Rapprich, V. 2012. Sedimentary record of subaerial volcanic activity in the basal Ordovician shoal-marine deposits: the Trrenice Formation of the Prague Basin, Bohemian Massif, Czech Republic. Bulletin of Geosciences 87(2), 359-372.

Jahn, J. J. 1904a. O krušnohorských vrstvách $\left(\mathrm{d}_{1 \alpha}\right)$ Rozpravy České Akademie pro vědy, slovesnost a umění, Tř́da II 13, 30, 1-16.

Jahn, J. J. 1904b. Über die Brachiopodenfauna der Bande $\mathrm{D}_{1}$. Verhandlungen der kaiserlich-königlichen geologischen Reichsanstalt 1904, 12, 270 280.

Katzer, F. 1892. Geologie von Böhmen. Der geognostische Aufbau und die geologische Entwickelung des Landes. Mit besonderer Berücksichtigung der Erzvorkommen und der verwendbaren Minerale und Gesteine. 1606 pp. I. Taussig, Prag. Klouček, C. 1915. Novinky z krušnohorských vrstev $-\mathrm{d}_{1 \alpha}$. Rozpravy České Akademie pro vědy, slovesnost a umění, Tř́lda II 24, 42, 1-3.

Klouček, C. 1917. Novinky z krušnohorských vrstev $-\mathrm{d}_{1 \alpha}$. Část III. Rozpravy České akademie cisaře Františka Josefa pro vědy slovesnost a umění, Tř́da II 26, 42, 1-4.

Klouček, C. 1919. Novinky z krušnohorských vrstev $-\mathrm{d}_{1 \alpha}$. Část IV. Rozpravy České Akademie věd a umění, Tř́da II 27, 38, 1-6.

Klouček, C. 1920a. Novinky z krušnohorských vrstev $-\mathrm{d}_{1 \alpha}$. Část V. Rozpravy České akademie věd a uméní, Tř́da II 29, 3, 1-4.
Klouček, C. 1920b. Nové nálezy fauny z vrstev krušnohorských, komárovských a ze středního kambria. Časopis Musea Království Českého, oddíl př́rodovědný 94, 122-123.

Klouček, C. 1922a. Objev fauny Euloma-Niobe u Ouval. Rozpravy České akademie věd a umění, Tř́la II 31, 5, 1-3.

Klouček, C. 1922b. Découverte de la faune EulomaNiobe près de Ouvaly (Bohême). Bulletin international de l'Académie des Sciences de Bohême 1922, 1-2.

Klouček, C. 1924. Nové objevy ve vrstvách krušnohorských $\mathrm{d}_{\alpha}\left(\operatorname{Dd}_{1 \alpha}\right)$. Rozpravy České Akademie věd a umění, Tř́da II 33, 26, 1-3.

Klouček, C. 1925. Nové objevy ve vrstvách krušnohorských $\mathrm{d}_{\alpha}$. Část II. Rozpravy České Akademie věd a umění, Tř́da II 34, 30, 1-3.

Klouček, C. 1927a. Několik kritických poznámek k práci p. ing. R. Růžičky: Fauna vrstev Eulomových rudního ložiska u Holoubkova (v Ouzkém). Věstník Státního geologického ústavu Československé republiky 3(4-5), 160-163.

Klouček, C. 1927b. Quelques remarques critiques à propos du travail de M. R. Růžička: La faune des couches à Euloma du gîte minéral prés de Holoubkov (à Ouzký). Věstník Státního geologického ústavu Československé republiky 3(4-5), 163-165.

Klouček, C. 1931a. Novější zprávy z českého Tremadoku $-\mathrm{d}_{\alpha}$. Věstník Státního geologického ústavu Československé republiky 7(1), 56-57.

Klouček, C. 1931b. Nouvelles données sur le Trémadoc de la Bohême. Věstník Státního geologického ústavu Československé republiky 7(1), 5758.

Koliha, J. 1918. Brachiopoda z krušnohorských vrstev $-\mathrm{d}_{1 \alpha}$. Časopis Musea Království českého 92, 128-139.

Koliha, J. 1924. Atremata z krušnohorských vrstev $\left(\mathrm{d}_{\alpha}\right)$. Palaeontographica Bohemiae 10, $1-61$.

Koliha, J. 1926a. Balticko-polská facie spodního ordoviku v Čechách. Věstník Státního geologického ústavu Československé republiky 2(4-6), 305-316.

Koliha, J. 1926b. Facies baltico-polonais de l'Ordovicien inférieur en Bohême. Věstník Státního geologického ústavu Československé republiky 2(4-6), 317-328. 
Koliha, J. 1930a. Nález tremadoku na Přeloučsku. Věstník Státního geologického ústavu Československé republiky 6(2), 65-66.

Koliha, J. 1930b. Sur la découverte du Trémadoc dans la région de Přelouč. Věstník Státního geologického ústavu Československé republiky 6(2), 66-67.

Koliha, J. 1937. Sur le Trémadocien et sur l'Arénigien inférieur en Bohême. Bulletin de la Société géologique de France, 5e série 7, 477-495.

Kraft, J. 1975. Dendroid graptolites of the Ordovician of Bohemia. Sborník Národního muzea v Praze, Oddíl př́rodovědný 31(3-5), 211-238.

Kraft, J. 1997. Zástupci třídy Graptolithina Bronn, $1846 v$ třenickém - dobrotivském souvrství pražské pánve (ordovik, barrandien). $168 \mathrm{pp}$. and Dodatek 79 pp. Habilitation thesis, Charles University, Prague, Czech Republic.

Kraft, V. 1928. Geologické poměry Rokycanska. Rokycany, 120 pp.

Krejčí, J. \& Feistmantel, K. 1890. Orografický a geotektonický přehled území silurského ve středních Čechách. Archiv pro př́rodovědecké prozkoumání Čech 5, 5. 1-94.

Krejčí, J. \& Helmhacker, R. 1879. Erläuterungen zur geologischen Karte der Umgebungen von Prag. Archiv der naturwissenschaftliche Landesdurchforschung von Böhmen 4, 2. Geologické oddělení. 1-175.

Krejčí, J. \& Helmhacker, R. 1885. Vysvětlení geologické mapy okolí pražského. Archiv přírodovědeckého výzkumu Čech 4, 2. Geologické oddělení. 1-136.

Kukal, Z. 1961. Composition and Origin of the Ordovician Sediments of the Třenice- and Mílina Beds (Conglomerates, Sandstones, Cherts Paleozoic of Central Bohemia). Sborník Ústředního ústavu geologického 28, 265-307. (In Czech, English summary.)

Lehnert, O., Fatka, O., Kraft, P., Frýda, J., Černý, P. \& Mařík, K. 2004. Tremadocian Fe stromatolites of the Prague Basin (Czech Republic): The oldest record of hydrothermal vent communities, 152-153. In Reitner, J., Reich, M. \& Schmidt, G. (eds) Geobilogie. 74. Jahrestagung der Paläontologischen Gesellschaft, Göttingen, 02. bis 08. Oktober 2004. Kurzfassungen der Vorträge und Poster.

Lipold, M. V. 1863. Die Eisensteilager der silurischen Grauwackenformation in Böhmen. Jahrbuch der kaiserlich-königlichen geologischen Reichsanstalt 13, 339-448.

Mergl, M. 1981. The genus Orbithele (Brachiopoda, Inarticulata) from the Lower Ordovician of Bohemia and Morocco. Věstník Ústředního ústavu geologického 56(5), 287-292.

Mergl, M. 1984a. Marcusodictyon, an encrusting bryozoan from the Lower Ordovician (Tremadocian) of Bohemia. Věstník Českého geologického ústavu 59(3), 171-172.

Mergl, M. 1984b. Fauna of the Upper Tremadocian of Central Bohemia. Sborník geologických věd, Paleontologie 26, 9-49.

Mergl, M. 1994a. Inarticulate brachiopod genera Elkania Ford and Elkanisca Havlíček in the Lower Ordovician of Bohemia. Věstník Českého geologického ústavu 69, 4, 47-55.

Mergl, M. 1994b. Trilobite fauna from the Třenice Formation (Tremadoc) in Central Bohemia. Folia Musei rerum naturalium Bohemiae occidentalis, Geologica 39, 1-31.

Mergl, M. 1996. Tafonomická ztráta informací příklad z barrandienského tremadoku (Taphonomic loss of information: example from Tremadoc of the Barrandian; In Czech). Seminár k 75. výroči narození Prof. RNDr. Bohuslava Růžičky. Sborník referátů. VŠB - Technická univerzita $v$ Ostravě, Institut geologického inženýrství. Ostrava, 14.

Mergl, M. 1997a. Obolid brachiopods with burrowing sculptures in the Lower Ordovician of Bohemia. Bulletin of the Czech Geological Survey 72 (2), 127-139.

Mergl, M. 1997b. Distribution of the lingulate brachiopod Thysanotos in Central Europe. Věstník Českého geologického ústavu 72(1), 27-35.

Mergl, M. 1997c. Selective dissolution of fossils an example from the Tremadoc of Bohemia. Sborník vědeckých prací VŠB - Technická univerzita $v$ Ostravě, Řada hornicko-geologická, zuláštní číslo, 13-17.

Mergl, M. 2002. Linguliformean and craniiformean brachiopods of the Ordovician (Třenice to Dobrotivá Formations) of the Barrandian Bohemia. Acta Musei Nationalis Pragae, Series B, Historia Naturalis 58(1-2), 1-82.

Mergl, M. 2006. Tremadocian Trilobites of the Prague Basin, Czech Republic. Acta Musei Pragae, Series B, Historia Naturalis 62(1-2), $1-70$. 
Mergl, M. 2009. Sukcese fosilních asociací třenického souvrství (tremadoc, ordovik) u Holoubkova (pražská pánev). Zprávy o geologických výzkumech $v$ roce 2008, 101-103.

Mergl, M. 2011. Reassessment of the Ordovician brachiopod Poramborthis and Poramborthidae. Memoirs of the Association of Australasian Palaeontologists 41, 351-358.

Mergl, M. 2012 Fosilní fauna třenického souvrství (ordovik, tremadok) u Cheznovic (jihozápadní část Barrandienu). Zprávy o geologických výzkumech $v$ roce $2011,139-143$.

Mergl, M. \& Prokop, R. J. 2006. Lower Ordovician cystoids (Rhombifera, Diploporita) from the Prague Basin (Czech Republic). Bulletin of Geosciences 81(1), 1-15.

Mikuláš, R. 2001. Nález ichnofosilií v třenickém souvrství (tremadok, ordovik Barrandienu) od Libečova. Zprávy o geologických výzkumech $v$ roce 2000, 39-40.

Prantl, F. \& Pribyl, A. 1949a. On the genus Symphysurus Goldfuss and allied forms from the Ordovician of Bohemia (Trilobitae). Věstník Královské české společnosti nauk, tř́da matematicko-přirodovědecká, 1948, 12, 1-16.

Prantl, F. \& Přibyl, A. 1949b. O českých tremadockých dendroidech. Rozpravy České akademie věd a umění, Tř́da II (matematicko-prrírodovědecká), 59, 20, 1-11.

Prantl, F. \& Růžička, R. 1941. Fauna spodního tremadoku Železných Hor. Rozpravy České akademie věd a umění, Třída II (matematicko-přirodovědecká), 51, 13, 1-36.

Přibyl, A. \& Vaněk, J. 1969. Über einige Trilobiten des mittelböhmischen Ordoviziums. Věstník Českého geologického ústavu 44(6), 365-374.

Prokop, R. J. 1964. Sphaeronitoidea Neumayr of the Lower Paleozoic of Bohemia. Sborník geologických věd, Paleontologie 3, 7-37.

Růžička, R. 1926. Fauna vrstev Eulomových rudního ložiska u Holoubkova (v Ouzkém). Část I.
Trilobiti. Rozpravy České akademie věd a umění 35, 39, 1-26.

Růžička, R. 1927. Fauna vrstev Eulomových rudního ložiska u Holoubkova (v Ouzkém). Č́st II. Rozpravy České akademie věd a umění 36, 60, 1-21.

Růžička, R. 1931. Doplňky k fauně vrstev Eulomových rudního ložiska u Holoubkova. Věstník Státního geologického ústavu Československé republiky 7(4-5), 387-406.

Vála, J. \& Helmhacker, R. 1872. Rudy železné v krajině mezi Prahou a Berounem. Archiv pro přirodovědecký výzkum Čech 2, 1, 87-336.

Vála, J. \& Helmhacker, R. 1874. Das Eisensteinvorkommen in der Gegend zwischen Prag und Beraun. Archiv der naturwissenschaftliche Landesdurchforschung von Böhmen 2, 1, 102407.

Vaněk, J. 1959. Čeled’ Lichaidae Hawle et Corda, 1847 ze středočeského staršího paleozoika (Trilobitae). Bohemia centralis, A - Scientiae naturales $1(3), 81-168$.

Vaněk, J. 1965. Die Trilobiten des mittelböhmischen Tremadoc. Senckenbergiana lethaea 46(4/6), 263-308.

Vaněk, J. 1999. Ordovician in the eastermost part of the Prague Basin (Úvaly and Brandýs areas) and its comparison with the Rokycany area (westernmost part of the basin). Palaeontologia Bohemiae 5(2), 5-20.

Waagen, W. 1887. Systême silurien du centre de la Bohême par Joachim Barrande. $1^{\text {ere }}$ Partie: Recherches Paléontologiques. Continuation éditeé par le Musée Bohême. Vol. 7. Classe des Echinodermes. Ordre des Cystidés. Texte et 39 Planches. 233 pp. Leipsic and Prague.

Woldřich, J. 1916. Geologické poměry v úvodí Kačáku mezi Unhoští a Nenačovicemi. Rozpravy České Akademie pro vědy, slovesnost a umění, Tř́da II 25, 37, 1-36. 